\title{
Year Class Coexistence or Competitive Exclusion for Strict Biennials?
}

\author{
N. V. Davydova, O. Diekmann, S. A. van Gils
}

\begin{abstract}
We consider a discrete time model of semelparous biennial population dynamics. Interactions between individuals are modelled with the aid of an "environmental" variable $I$. The impact on and the sensitivity to the environmental condition is age specific. The main result is that competitive exclusion between the year classes is possible as is their coexistence. For moderate values of the basic reproduction ratio $R_{0}$ there is a strict dichotomy: depending on the other parameters we either find competitive exclusion or coexistence. We characterize rather precisely the patterns of age specific impact and sensitivity that lead to either of these outcomes.
\end{abstract}

Key words: competitive exclusion, semelparous species, periodical insects.

\section{Introduction.}

If individuals can reproduce only once in their life, the species is called semelparous or, in the case of insects, univoltine. Examples range from annual and biennial plants to salmon to cicadas.

If reproduction is restricted to a small time window in the year and life span has a fixed length of, say, $k$ years, a population splits into year classes according to the year of birth (counted modulo $k$, which we write as $(\bmod k))$ or, equivalently, the year of reproduction $(\bmod k)$. (Note the terminology: an individual belongs to the same year class throughout its life, whereas the age class to which it belongs is determined by its age and therefore increases by one each year; we shall number the age classes from 0 to $k-1$.) As a year class is reproductively isolated from the other year classes, it forms a population by itself.

Yet year classes are likely to interact, for instance by competition for food. It may then happen that a year class is driven to extinction. Bulmer [3] calls an insect periodical if it consists of a single year class, i.e. if all but one year classes are missing. Famous examples are the cicada species with 13 and 17 year life cycles (see e.g. [2] and the references in there).

Mathematically the phenomenon of possibly missing year classes is showing up as invariance of coordinate axes and (hyper)planes for the "full life cycle" map of looking $k$ years ahead. For each invariant subspace we can discuss/investigate the dynamics within it, as well as the transversal stability, by which we mean the attraction or repulsion in the transverse direction (or, in more biological terms, the decline or growth of a missing year class which is introduced in small numbers). 
We shall describe interaction (i.e. density dependence) as feedback via the environment (see the next section for details and see [9] for the general philosophy). The phenomenon of periodical insects then leads to the following questions in the context of a model: can one year class tune the environmental conditions such that the other year classes are driven to extinction when rare? Or can missing year classes invade successfully? Do we get coexistence or competitive exclusion?

In this paper we shall derive a rather complete answer to these questions under two rather restrictive assumptions. The first is that we shall take $k=2$, i.e. we focus on strict biennials. The second is that we assume that the environmental conditions are fully characterised by a scalar quantity, which we denote by the symbol $I$ (the letter " I" comes from the word "input" and means environmental input to behaviour of individuals).

The model of Bulmer [3] is specified in terms of the quantities $m_{i j}$ which measure the influence of the presence of $j$ year old individuals on the survival (or, in the case $i=k-1$, also reproduction) of $i$ year old individuals. Here we consider the special case $m_{i j}=g_{i} c_{j}$ (so the matrix $m$ has one-dimensional range spanned by the vector $g$ ). The components of $g$ then correspond to the age-specific sensitivity to environmental conditions and the components of $c$ to the age-specific impact on the environmental conditions.

By numerical experimentation Bulmer [3] arrives at the following conclusion: competitive exclusion prevails if competition is more severe between than within age classes (i.e., the off-diagonal elements of the matrix $m$ are bigger than the diagonal elements). Our analytical results for the special case allow a somewhat different conclusion: competitive exclusion prevails if the sensitivity increases with age while the impact decreases with age and, also, if the sensitivity decreases with age while the impact increases sufficiently strongly with age (see Theorem 10.2).

It is possible to extend this conclusion to arbitrary values of $k$ [1] and we intend to deal with higher values of $k$ in future papers. The reason to restrict to $k=2$ here is that this case allows a rather complete analysis with only simple tools. Wherever appropriate, however, we shall choose our notation and formulate our results in such a way that it will easily extend to general values of $k$. (Thus we hope to avoid redundant repetition.)

In work with S. Mylius [10] one of us has analysed the special case of nursery competition (only $c_{k-1}$ and $g_{k-1}$ are different from zero). In that special case the interaction is restricted to the own year class and, as a consequence, we simply deal with $k$ copies of one and the same discrete time dynamical system. The classification of all (essentially different, i.e. unrelated by time translation) periodic patterns that arise is presented in [8]. The papers $[10,13]$ deal with the competition between species with different values of $k$. Key words are "resonance mediated coexistence" and "invasible yet invincible strategy" (or "the resident strikes back").

The main, from a biological point of view, conclusions of this paper are presented in Section 10. They take the form of a clear-cut alternative: for not too high values of the basic reproduction ratio $R_{0}$, either the two year classes coexist in steady state or one is missing and the other is steady in every phase of its life, so performs a two-cycle. We characterize precisely the parameter combinations that lead to either of these alternatives and, in addition, interpret these parameter conditions biologically.

There exists a wealth of literature on models that differ only slightly from the one considered here. We refer to Nisbet \& Onyiah [14] and Wikan [15] and the references given therein. 


\section{The model formulation.}

In this paper we analyse the nonlinear Leslie matrix iteration

$$
N(t+1)=L(I(t)) N(t),
$$

where

$$
N(t)=\left(\begin{array}{c}
N_{0}(t) \\
N_{1}(t)
\end{array}\right),
$$

so the components of $N(t)$ measure the size of the various age classes at time $t$, and

$$
\begin{gathered}
L(I)=\sqrt{R_{0}}\left(\begin{array}{cc}
0 & e^{-g_{1} I} \\
e^{-g_{0} I} & 0
\end{array}\right), \\
I=c \cdot N=c_{0} N_{0}+c_{1} N_{1}
\end{gathered}
$$

with normalization of parameters such that

$$
\begin{aligned}
& g_{0}+g_{1}=1, \\
& c_{0}+c_{1}=1 .
\end{aligned}
$$

Our aim is to derive how the qualitative dynamical behaviour depends on the three parameters $R_{0}, c_{0}$ and $g_{0}$.

The formulation above is obtained by scaling the equations

$$
\begin{aligned}
& \tilde{N}_{0}(t+1)=f s_{1} e^{-\tilde{g_{1}} \tilde{I}(t)} \tilde{N}_{1}(t) \\
& \tilde{N}_{1}(t+1)=s_{0} e^{-\tilde{g_{0}} \tilde{I}(t)} \tilde{N}_{0}(t),
\end{aligned}
$$

where $s_{0}$ and $s_{1}$ are survival probabilities under "ideal" conditions, $f$ is the expected number of offspring of a reproducing individual (again under "ideal" conditions), $R_{0}=s_{0} s_{1} f$ and $\exp \left(-\tilde{g_{0}} \tilde{I}(t)\right)$ is the reduction of the survival probability in the first year due to crowding, while $\exp \left(-\tilde{g_{1}} \tilde{I}(t)\right)$ measures the reduction (due to crowding) of the product of the survival probability in the second year and the expected number of offspring of a reproducing individual. We do not specify what exactly are the adverse effects of crowding (they may be a reduction of the food (or light or territorium) availability or an increase of the concentration of toxic substances or the density of predators). Instead we simply postulate that their effect is described well by the factors $\exp \left(-\tilde{g_{0}} \tilde{I}(t)\right)$ and $\exp \left(-\tilde{g_{1}} \tilde{I}(t)\right)$ with $\tilde{I}(t)$ such that

$$
\tilde{I}(t)=\tilde{c_{0}} \tilde{N}_{0}(t)+\tilde{c_{1}} \tilde{N}_{1}(t) .
$$

Explicitly the scaling is given by

$$
\begin{aligned}
& \tilde{N}_{0}(t)=\sqrt{f s_{1}} \frac{N_{0}(t)}{\left(\tilde{g_{0}}+\tilde{g_{1}}\right)\left(\tilde{c_{0}} \sqrt{f s_{1}}+\tilde{c_{1}} \sqrt{s_{0}}\right)}, \\
& \tilde{N}_{1}(t)=\sqrt{s_{0}} \frac{N_{1}(t)}{\left(\tilde{g_{0}}+\tilde{g_{1}}\right)\left(\tilde{c_{0}} \sqrt{f s_{1}}+\tilde{c_{1}} \sqrt{s_{0}}\right)}, \\
& g_{0}=\frac{\tilde{g_{0}}}{\tilde{g_{0}}+\tilde{g_{1}}}, \quad g_{1}=\frac{\tilde{g_{1}}}{\tilde{g_{0}}+\tilde{g_{1}}} \\
& c_{0}=\frac{\tilde{c_{0}} \sqrt{f s_{1}}}{\tilde{c_{0}} \sqrt{f s_{1}}+\tilde{c_{1}} \sqrt{s_{0}}}, \quad c_{1}=\frac{\tilde{c_{1}} \sqrt{s_{0}}}{\tilde{c_{0}} \sqrt{f s_{1}}+\tilde{c_{1}} \sqrt{s_{0}}} \text {, }
\end{aligned}
$$




$$
I(t)=\left(\tilde{g_{0}}+\tilde{g_{1}}\right) \tilde{I}(t)
$$

The advantage of the form (2.3) compared to alternatives obtained by slightly different scalings, is that a certain parameter symmetry finds a rather simple expression.

Before embarking upon the analysis we uncover this symmetry in the parameter space which derives from a combination of two special features:

- life is a cycle and, as the difference between survival and reproduction is not reflected in the mathematical structure, we might as well shift the order in which we list the age classes;

- the reduction factors have the same functional form and differ only in a parameter value.

Proposition 2.1. For fixed $R_{0}$, the dynamics do not change if we interchange both $c_{0}$ and $c_{1}$ as well as $g_{0}$ and $g_{1}$ (or, in other words, replace $c_{0}$ by $1-c_{0}$ and $g_{0}$ by $\left.1-g_{0}\right)$.

Proof. If we introduce the notation

$$
\begin{aligned}
& S\left(\begin{array}{l}
N_{0} \\
N_{1}
\end{array}\right)=\left(\begin{array}{l}
N_{1} \\
N_{0}
\end{array}\right), \\
& R\left(\begin{array}{c}
c_{0} \\
g_{0}
\end{array}\right)=\left(\begin{array}{l}
1-c_{0} \\
1-g_{0}
\end{array}\right), \\
& F\left(N,\left(\begin{array}{c}
c_{0} \\
g_{0}
\end{array}\right)\right)=L_{g_{0}}\left(c_{0} N_{0}+\left(1-c_{0}\right) N_{1}\right) N
\end{aligned}
$$

with

$$
L_{g_{0}}(I)=\sqrt{R_{0}}\left(\begin{array}{cc}
0 & e^{-g_{0} I} \\
e^{-\left(1-g_{0}\right) I} & 0
\end{array}\right),
$$

then, as one can easily verify,

$$
S F\left(S^{-1} x,\left(\begin{array}{c}
c_{0} \\
g_{0}
\end{array}\right)\right)=F\left(x, R\left(\begin{array}{l}
c_{0} \\
g_{0}
\end{array}\right)\right),
$$

so the transformation $S^{-1}$ of the state variables is exactly "neutralised" by the transformation $R$ of the parameters.

Note that both $R$ and $S$ are reflections and that, for given $R_{0}$, we need only to investigate half of the parameter square $\left\{\left(\begin{array}{c}c_{0} \\ g_{0}\end{array}\right): 0 \leq c_{0} \leq 1,0 \leq g_{0} \leq 1\right\}$. (For higher values of $k$ one should replace $R$ and $S$ by the generator of the cyclic group of order $k$.) 


\section{Steady coexistence of the year classes.}

Before providing precise formulations and detailed derivations we give a sectionsummary in one sentence: there is a unique steady state (3.1) and the region in parameter space corresponding to its stability is described completely in Proposition 3.6. Readers uninterested in technical details can jump to the end of the section for a somewhat more detailed summary.

Theorem 3.1. Let $R_{0}>1$.

i) There exists a unique nontrivial steady state given explicitly by

$$
\bar{N}=\left(\begin{array}{c}
\bar{N}_{0} \\
\bar{N}_{1}
\end{array}\right)=\frac{\ln R_{0}}{c_{0}+\left(1-c_{0}\right) R_{0}^{\frac{1}{2}-g_{0}}}\left(\begin{array}{c}
1 \\
R_{0}^{\frac{1}{2}-g_{0}}
\end{array}\right) .
$$

The corresponding value of the environmental condition is $\bar{I}=\ln R_{0}$.

ii) This steady state is locally asymptotically stable whenever both the inequalities

$$
\begin{gathered}
\left(g_{0}-\frac{1}{2}\right)\left(c_{0}-\frac{R_{0}^{\frac{1}{2}-g_{0}}}{1+R_{0}^{\frac{1}{2}-g_{0}}}\right)>0 \\
c_{0}\left(g_{0} \ln R_{0}-2+\left(2+\left(g_{0}-1\right) \ln R_{0}\right) R_{0}^{\frac{1}{2}-g_{0}}\right)<\left(2+\left(g_{0}-1\right) \ln R_{0}\right) R_{0}^{\frac{1}{2}-g_{0}}
\end{gathered}
$$

hold, and unstable if at least one of these inequalities is reversed.

Proof. If the system is in steady state, the variable $I$ is constant, which we denote by $\bar{I}$, and the dominant eigenvalue $\left(R_{0} e^{-\bar{I}}\right)^{\frac{1}{2}}$ of $L(\bar{I})$ should equal one. So $\bar{I}=$ $\ln R_{0}$ is the only possibility. The corresponding eigenvector $\bar{N}$, normalised such that $c \cdot \bar{N}=\bar{I}$, is given by (3.1).

Let us now concentrate on (ii). We put $N(t)=\bar{N}+x(t)$ and, correspondingly, $I(t)=\bar{I}+c \cdot x(t)$, while assuming that $x$ is small. Inserting this into (2.1) we find

$$
x(t+1)=L(\bar{I}) x(t)+c . x(t) D L(\bar{I}) \bar{N}+\text { h.o.t. }
$$

where $D L(I)$ is represented by the matrix

$$
\left(\begin{array}{cc}
0 & -g_{1} \sqrt{R_{0}} e^{-g_{1} I} \\
-g_{0} \sqrt{R_{0}} e^{-g_{0} I} & 0
\end{array}\right)
$$

and consequently

$$
D L(\bar{I}) \bar{N}=-\frac{\ln R_{0}}{c_{0}+\left(1-c_{0}\right) R_{0}^{\frac{1}{2}-g_{0}}}\left(\begin{array}{c}
1-g_{0} \\
g_{0} R_{0}^{\frac{1}{2}-g_{0}}
\end{array}\right)=-\bar{N}_{0}\left(\begin{array}{c}
1-g_{0} \\
g_{0} R_{0}^{\frac{1}{2}-g_{0}}
\end{array}\right) .
$$

By deleting the h.o.t. we arrive at the linearised problem

$$
x(t+1)=M x(t)
$$

with the matrix $M$ given explicitly by

$$
M=\left(\begin{array}{cc}
-c_{0} g_{1} \bar{N}_{0} & R_{0}^{\frac{1}{2}-g_{1}}-c_{1} g_{1} \bar{N}_{0} \\
R_{0}^{\frac{1}{2}-g_{0}}\left(1-c_{0} g_{0} \bar{N}_{0}\right) & -c_{1} g_{0} \bar{N}_{0} R_{0}^{\frac{1}{2}-g_{0}}
\end{array}\right)
$$




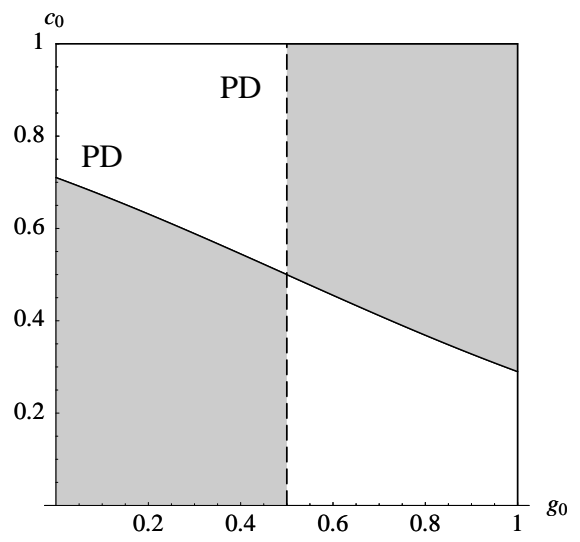

Figure 1: The regions in the $\left(g_{0}, c_{0}\right)$-square where the condition (3.2) holds are shaded. $R_{0}=6<e^{2}$. The internal boundary is formed by two PD-curves.

so that

$$
\begin{aligned}
& T=\operatorname{trace} M=-\left(g_{1} c_{0}+g_{0} c_{1} R_{0}^{\frac{1}{2}-g_{0}}\right) \bar{N}_{0} \\
& D=\operatorname{det} M=-1+\left(c_{0} g_{0}+c_{1} g_{1} R_{0}^{\frac{1}{2}-g_{0}}\right) \bar{N}_{0} .
\end{aligned}
$$

The three conditions $D<1, T<D+1, T>-D-1$ guarantee stability and whenever the opposite of at least one of the three holds we have instability. As to be expected on the basis of the uniqueness of the nontrivial steady state, the condition $T<D+1$ always holds (since $D+1 \geq 0$ while $T \leq 0$ and the possibility that $T=D+1=0$ is readily excluded; note that the equality $T=D+1$ corresponds to an eigenvalue being +1 ). The condition $T>-D-1$ can be rewritten in the form (3.2) and the condition $D<1$ in the form (3.3).

Equality in the condition (3.2) corresponds to a period-doubling bifurcation, while equality in (3.3) corresponds to a Neimark-Sacker bifurcation, i.e. a Hopf bifurcation for maps (see, for example, [11]).

It is convenient to give a graphical representation of the two stability condition (3.2) and (3.3) in the $\left(g_{0}, c_{0}\right)$-square, for various values of $R_{0}$ and the rest of this section is devoted to doing just that. The two factors at the left-hand side of (3.2) determine two curves, $g_{0}=\frac{1}{2}$ and $c_{0}=\frac{R_{0}^{\frac{1}{2}-g_{0}}}{1+R_{0}^{\frac{1}{2}-g_{0}}}$ that cross each other at $\left(\frac{1}{2}, \frac{1}{2}\right)$. (We shall call these PD-curves, for "period-doubling". Note that indeed the curve $c_{0}=\frac{R_{0}^{\frac{1}{2}-g_{0}}}{1+R_{0}^{\frac{1}{2}-g_{0}}}$ is invariant under the transformation $\left(g_{0}, c_{0}\right) \mapsto\left(1-g_{0}, 1-c_{0}\right)$ as "predicted" by Proposition 2.1.) The condition (3.2) holds in the North-East and in the South-West quadrant of the partitioning of the square by the two curves (Fig. 1).

Next, let us turn our attention to (3.3). The symmetry (Proposition 2.1) allows us to focus on $0 \leq g_{0} \leq \frac{1}{2}$. We want to rewrite (3.3) in the form $c_{0}>\psi\left(g_{0}, R_{0}\right)$, but to do so we need information about the sign of the second factor at the left-hand side of (3.3). For ease of formulation we give the name $\phi$ to the product of $R_{0}^{g_{0}-\frac{1}{2}}$ and this factor:

$$
\phi\left(g_{0}, R_{0}\right)=\left(g_{0} \ln R_{0}-2\right) R_{0}^{g_{0}-\frac{1}{2}}+2+\left(g_{0}-1\right) \ln R_{0} .
$$


Lemma 3.2. Let $R_{0}>1$. Then $\phi\left(g_{0}, R_{0}\right)<0$ for $0 \leq g_{0}<\frac{1}{2}$ and $\phi\left(\frac{1}{2}, R_{0}\right)=0$.

Proof. Clearly $\phi\left(\frac{1}{2}, R_{0}\right)=0$. Furthermore, $\phi\left(g_{0}, 1\right)=0$ and

$$
\begin{aligned}
\frac{\partial \phi}{\partial R_{0}}\left(g_{0}, R_{0}\right) & =g_{0} R_{0}^{g_{0}-\frac{3}{2}}+\left(g_{0} \ln R_{0}-2\right)\left(g_{0}-\frac{1}{2}\right) R_{0}^{g_{0}-\frac{3}{2}}+\left(g_{0}-1\right) R_{0}^{-1} \\
& =\left(1-g_{0}\right)\left(R_{0}^{g_{0}-\frac{3}{2}}-R_{0}^{-1}\right)+\left(g_{0}-\frac{1}{2}\right) g_{0} R_{0}^{g_{0}-\frac{3}{2}} \ln R_{0}
\end{aligned}
$$

For $R_{0}>1$ and $0 \leq g_{0}<\frac{1}{2}$ both these last two terms are negative.

Proposition 3.3. For $R_{0}>1$ and $0 \leq g_{0}<\frac{1}{2}$ the inequality (3.3) is equivalent to

$$
c_{0}>\psi\left(g_{0}, R_{0}\right)
$$

where by definition

$$
\psi\left(g_{0}, R_{0}\right)=\frac{2+\left(g_{0}-1\right) \ln R_{0}}{\phi\left(g_{0}, R_{0}\right)} .
$$

The curve $c_{0}=\psi\left(g_{0}, R_{0}\right)$ for a fixed $R_{0}$ corresponds to a Neimark-Sacker bifurcation. Accordingly, we will call this a NS-curve.

\section{Lemma 3.4.}

i) For $1<R_{0}<e^{4}$ and $0 \leq g_{0}<\frac{1}{2}$ the inequality

$$
\frac{\partial \psi}{\partial g_{0}}\left(g_{0}, R_{0}\right)<0
$$

holds.

ii)

$$
\psi\left(g_{0}, e^{4}\right)=\frac{e^{4\left(\frac{1}{2}-g_{0}\right)}}{1+e^{4\left(\frac{1}{2}-g_{0}\right)}},
$$

(so, in particular, $\psi\left(\cdot, e^{4}\right)$ has a continuous extension to the interval $[0,1]$ with $\left.\psi\left(\frac{1}{2}, e^{4}\right)=\frac{1}{2}\right)$.

iii) For $R_{0}>e^{4}$ define $\overline{g_{0}}=\overline{g_{0}}\left(R_{0}\right)=\frac{1}{2}-\sqrt{\frac{1}{4}-\frac{1}{\ln R_{0}}}$. Then

$$
\frac{\partial \psi}{\partial g_{0}}\left(g_{0}, R_{0}\right)<0 \quad \text { for } \quad R_{0}>e^{4} \quad \text { and } \quad 0 \leq g_{0}<\overline{g_{0}}
$$

while

$$
\frac{\partial \psi}{\partial g_{0}}\left(g_{0}, R_{0}\right)>0 \quad \text { for } \quad R_{0}>e^{4} \quad \text { and } \quad \overline{g_{0}}<g_{0}<\frac{1}{2} .
$$

Proof. From the definition (3.7) we deduce that

$$
\begin{aligned}
\operatorname{sign}\left(\frac{\partial \psi}{\partial g_{0}}\left(g_{0}, R_{0}\right)\right) & =\operatorname{sign}\left(\left\{\ln R_{0} \phi\left(g_{0}, R_{0}\right)-\left(2+\left(g_{0}-1\right) \ln R_{0}\right) \frac{\partial \phi}{\partial g_{0}}\left(g_{0}, R_{0}\right)\right\}\right) \\
& =\operatorname{sign}\left(\left(g_{0} \ln R_{0}-2-\left(2+\left(g_{0}-1\right) \ln R_{0}\right)\left(g_{0} \ln R_{0}-1\right)\right)\right) \\
& =\operatorname{sign}\left(\left(-1-g_{0}\left(g_{0}-1\right) \ln R_{0}\right)\right) .
\end{aligned}
$$

Since $-g_{0}\left(g_{0}-1\right) \leq \frac{1}{4}$ the assertion in i) follows at once. Furthermore, for $R_{0}>e^{4}$ the function $g_{0} \mapsto-1-g_{0}\left(g_{0}-1\right) \ln R_{0}$ switches sign, from negative to positive, at $\overline{g_{0}}$, whence the inequalities in iii) hold. Finally, the expression for $\psi\left(g_{0}, e^{4}\right)$ is derived by a straightforward computation. 


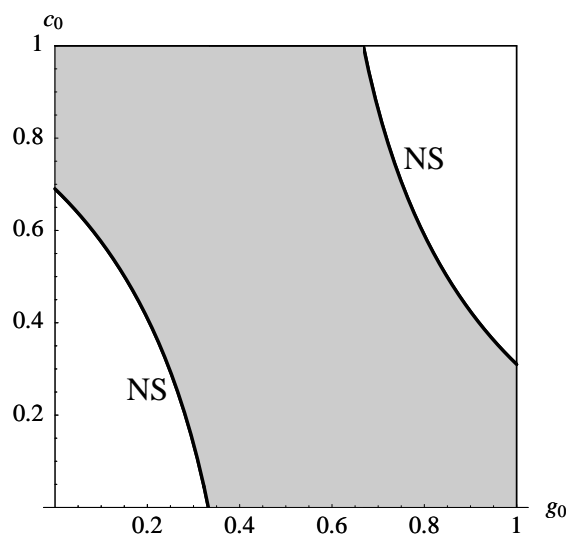

Figure 2: The region in the $\left(g_{0}, c_{0}\right)$-square where the condition (3.3) holds is shaded. $R_{0}=20<e^{4}$. The internal boundary is formed by two NS-curves.

\section{Proposition 3.5.}

i) For $1<R_{0}<e^{2}$, (3.3) is satisfied for all $\left(g_{0}, c_{0}\right)$.

ii) For $R_{0}=e^{2},(3.3)$ is satisfied for all $\left(g_{0}, c_{0}\right)$ except $(0,0)$ and $(1,1)$.

iii) For $e^{2}<R_{0}<e^{4}$, (3.3) is satisfied in a domain as depicted in Figure 2, the characteristic feature being that the left nontrivial boundary is the monotone decreasing curve

$$
c_{0}=\psi\left(g_{0}, R_{0}\right)
$$

connecting the points

$$
\left(0, \frac{\ln R_{0}-2}{\ln R_{0}-2+2 R_{0}^{-\frac{1}{2}}}\right)
$$

and

$$
\left(1-\frac{2}{\ln R_{0}}, 0\right)
$$

iv) For $R_{0}=e^{4}$, (3.3) is satisfied in the region

$$
c_{0}>\psi\left(g_{0}, e^{4}\right)=\frac{e^{4\left(\frac{1}{2}-g_{0}\right)}}{1+e^{4\left(\frac{1}{2}-g_{0}\right)}}, \quad 0 \leq g_{0}<\frac{1}{2},
$$

and its mirror image under the transformation $R$ introduced in (the proof of) Proposition 2.1 (Fig. 3).

v) For $R_{0}>e^{4}$, (3.3) is satisfied in two regions as depicted in Figure 4, the characteristic feature being that the boundary curve

$$
c_{0}=\psi\left(g_{0}, R_{0}\right)
$$

connects a point on $g_{0}=0$ with a point on $c_{0}=1$, starting off in a decreasing manner and then changing into an increasing curve.

Having understood (3.2) and (3.3) separately, we now superimpose the results.

\section{Proposition 3.6.}

i) For $R_{0}>e^{4}$ the steady state (3.1) is unstable. 


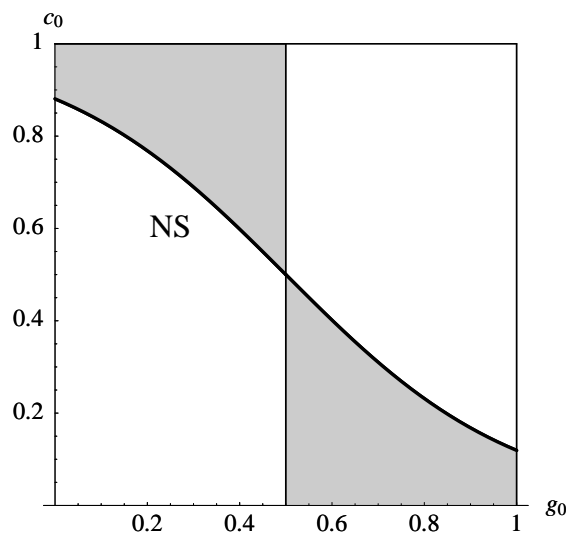

Figure 3: The regions in the $\left(g_{0}, c_{0}\right)$-square where the condition (3.3) holds are shaded. $R_{0}=e^{4}$. The internal boundary is formed by the NS-curve and by the line $g_{0}=\frac{1}{2}$ corresponding to a double eigenvalue -1 .

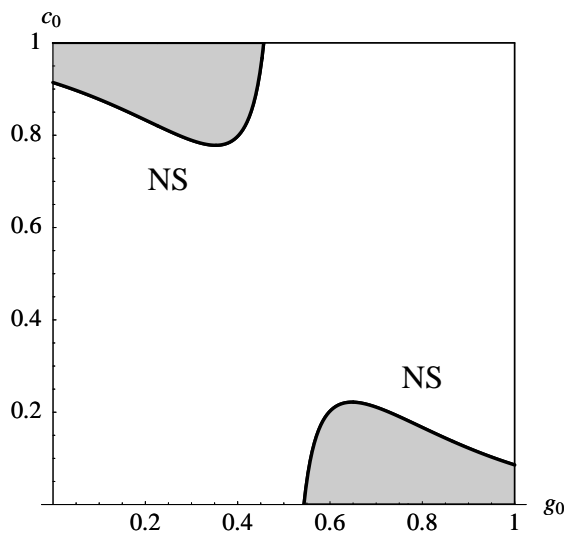

Figure 4: The regions in the $\left(g_{0}, c_{0}\right)$-square where the condition (3.3) holds are shaded. $R_{0}=80>e^{4}$. The internal boundary is formed by two NS-curves.

ii) For $R_{0}=e^{4}$ and either $g_{0}=\frac{1}{2}$ or $c_{0}=\frac{e^{4\left(\frac{1}{2}-g_{0}\right)}}{1+e^{4\left(\frac{1}{2}-g_{0}\right)}}$ there is a double eigenvalue -1 associated with the steady state (3.1). For $R_{0}=e^{4}$ and other $\left(g_{0}, c_{0}\right)$-values the steady state is unstable.

iii) For $e^{2} \leq R_{0}<e^{4}$ the steady state is stable in the parameter region

$$
\psi\left(g_{0}, R_{0}\right)<c_{0}<\frac{R_{0}^{\frac{1}{2}-g_{0}}}{1+R_{0}^{\frac{1}{2}-g_{0}}}, \quad 0 \leq g_{0}<\frac{1}{2}
$$

and its mirror image under the transformation $R$ of Proposition 2.1. For $g_{0}=\frac{1}{2}$ and for $c_{0}=\frac{R_{0}^{\frac{1}{2}-g_{0}}}{1+R_{0}^{\frac{1}{2}-g_{0}}}$ there is an eigenvalue -1 , while for $c_{0}=$ $\psi\left(g_{0}, R_{0}\right)$ there is a pair of complex eigenvalues on the unit circle. For all remaining $\left(g_{0}, c_{0}\right)$-values the steady state is unstable.

iv) For $1<R_{0}<e^{2}$ the steady state is stable in the parameter region

$$
c_{0}<\frac{R_{0}^{\frac{1}{2}-g_{0}}}{1+R_{0}^{\frac{1}{2}-g_{0}}}, \quad 0 \leq g_{0}<\frac{1}{2}
$$




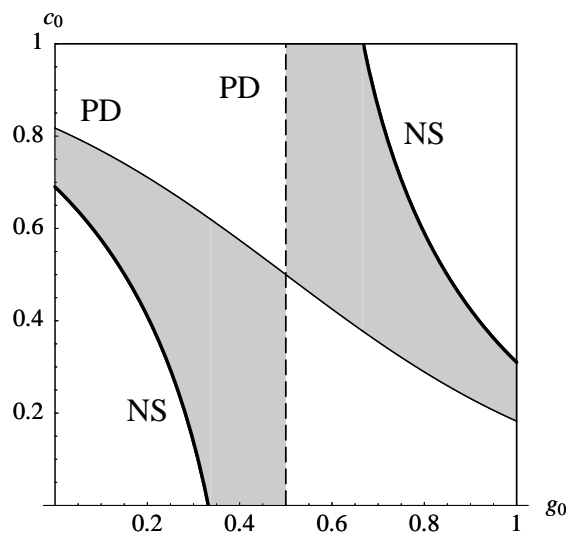

Figure 5: The regions of stability of the steady state are shaded. They are bounded by the two PD-curves and the two NS-curves. $e^{2}<R_{0}=20<e^{4}$.

and its mirror image under $R$. For $g_{0}=\frac{1}{2}$ and for $c_{0}=\frac{R_{0}^{\frac{1}{2}-g_{0}}}{1+R_{0}^{\frac{1}{2}-g_{0}}}$ there is an eigenvalue -1 . For all remaining $\left(g_{0}, c_{0}\right)$-values the steady state is unstable.

Proof.

i) The point is that for $R_{0}>e^{4}$ and $0 \leq g_{0}<\frac{1}{2}$ we have $\psi\left(g_{0}, R_{0}\right)>\frac{R_{0}^{\frac{1}{2}-g_{0}}}{1+R_{0}^{\frac{1}{2}-g_{0}}}$ (whence it is impossible to satisfy both (3.2) and (3.3)).

ii) For $R_{0}=e^{4}$ the two curves $c_{0}=\psi\left(g_{0}, R_{0}\right)$ and $c_{0}=\frac{R_{0}^{\frac{1}{2}-g_{0}}}{1+R_{0}^{\frac{1}{2}-g_{0}}}$ coincide exactly and, as one easily verifies, on this curve as well as on the line $g_{0}=\frac{1}{2}$ we have, for $T$ and $D$ defined in (3.4) that $T=-2, D=1$, so the characteristic equation then reads $0=\lambda^{2}-T \lambda+D=(\lambda+1)^{2}$.

iii) and iv) are simply combinations of earlier results.

Let us now summarize in words the results that we need in the sequel.

- For $R_{0}>1$ the map (2.1) has a unique nontrivial fixed point.

- If $1<R_{0}<e^{2}$ the stability regions of this steady state are bounded by the PD-curves and shown as shaded in Figure 1.

- If $e^{2}<R_{0}<e^{4}$ the stability regions are bounded by the PD-curves and the NS-curves as presented in Figure 5.

- If $R_{0}>e^{4}$ the steady state is unstable.

In the rest of the paper we will mainly deal with values of $R_{0}$ in the interval $\left(1, e^{4}\right)$.

\section{Environmental conditions of period one or two.}

The structure of (2.1) suggests the following method of analysis: 
- first assume a certain periodicity of $I$ and analyse the consequences for $N$;

- next verify whether the assumptions and consequences are compatible with the relation (2.4) between $I$ and $N$.

In particular, we will show in this section that generically,

- constant environmental conditions require that the system is in steady state;

- solutions with minimal period two and both year classes present do not exist.

The exceptional parameter combinations are pointed out explicitly. The study of the dynamics in these special cases is the subject of the sections 5 and 6 .

Theorem 4.1. Provided $c_{0} \neq \frac{R_{0}^{\frac{1}{2}-g_{0}}}{1+R_{0}^{\frac{1}{2}-g_{0}}}$, the environmental condition I is constant only if the system is in steady state.

Proof. When $I(t)=\bar{I}$ for all $t$, the recurrence relation (2.1) is linear. As neither exponential growth of $N(t)$ nor exponential decline is consistent with $I(t)=c \cdot N(t)$ being constant, we must have that the dominant eigenvalue of $L(\bar{I})$ equals one, i.e. that $\bar{I}=\ln R_{0}$ (see Theorem 3.1.i).

For this choice of $\bar{I}$, any initial condition $\left(\bar{N}_{0}, \bar{N}_{1}\right)^{T}$ leads to a 2-periodic orbit, the second point on the orbit being $\left(R_{0}^{-\frac{1}{2}+g_{0}} \bar{N}_{1}, R_{0}^{\frac{1}{2}-g_{0}} \bar{N}_{0}\right)^{T}$. In order for such an orbit to be consistent with our assumption that $I(t)=\ln R_{0}$ for all $t$, we should have that

$$
\begin{array}{ll}
c_{0} \bar{N}_{0}+c_{1} \bar{N}_{1} & =\ln R_{0} \\
c_{1} R_{0}^{\frac{1}{2}-g_{0}} \bar{N}_{0}+c_{0} R_{0}^{-\frac{1}{2}+g_{0}} \bar{N}_{1} & =\ln R_{0} .
\end{array}
$$

Provided the determinant $c_{0}^{2} R_{0}^{-\frac{1}{2}+g_{0}}-c_{1}^{2} R_{0}^{\frac{1}{2}-g_{0}}$ is non-zero we find a unique point $\bar{N}$ which is, in fact, given by (3.1). Since $c_{0}$ and $c_{1}$ are nonnegative, the condition on the determinant translates into $c_{0} \neq c_{1} R_{0}^{\frac{1}{2}-g_{0}}$.

Theorem 4.2. Let $R_{0}>1, g_{0} \neq \frac{1}{2}, c_{0} \neq \frac{R_{0}^{\frac{1}{2}-g_{0}}}{1+R_{0}^{\frac{1}{2}-g_{0}}}$. Assume that $I(t)$ is periodic with period two and that both year classes are present. Then, in fact, the system must be in the nontrivial steady state (and, correspondingly, $I(t)$ must be constant and therefore equal to $\bar{I}=\ln R_{0}$ ).

Proof. Let $I_{0}$ and $I_{1}$ denote the two values that the environmental condition alternatingly takes. Since

$$
L\left(I_{1}\right) L\left(I_{0}\right)=R_{0}\left(\begin{array}{cc}
e^{-g_{0} I_{0}-g_{1} I_{1}} & 0 \\
0 & e^{-g_{0} I_{1}-g_{1} I_{0}}
\end{array}\right)
$$

we must have that both $R_{0} e^{-g_{0} I_{0}-g_{1} I_{1}}$ and $R_{0} e^{-g_{0} I_{1}-g_{1} I_{0}}$ are equal to one, or, equivalently

$$
\left(\begin{array}{ll}
g_{0} & g_{1} \\
g_{1} & g_{0}
\end{array}\right)\left(\begin{array}{l}
I_{0} \\
I_{1}
\end{array}\right)=\ln R_{0}\left(\begin{array}{l}
1 \\
1
\end{array}\right)
$$

If $g_{0} \neq \frac{1}{2}$ we have $g_{0} \neq g_{1}$ and the matrix is invertible. Clearly the unique solution is $I_{0}=I_{1}=\ln R_{0}$ and from Theorem 4.1 we infer that the system is in the nontrivial steady state if both year classes are present and $c_{0} \neq \frac{R_{0}^{\frac{1}{2}-g_{0}}}{1+R_{0}^{\frac{1}{2}-g_{0}}}$. 


\section{The special case $g_{0}=\frac{1}{2}$ of "uniform sensi- tivity".}

For $g_{0}=\frac{1}{2}$ the two-dimensional map decomposes into a one-parameter family of one-dimensional maps. This observation reveals a branch of 2-cycles at $g_{0}=\frac{1}{2}$ and thus how the steady state (3.1) undergoes a "vertical" period-doubling bifurcation at $g_{0}=\frac{1}{2}$.

Theorem 5.1. Let $g_{0}=\frac{1}{2}$. In terms of polar coordinates, the map (2.1) is given by

$$
(r, \varphi) \mapsto\left(\sqrt{R_{0}} r e^{-\alpha(\varphi) r}, \frac{\pi}{2}-\varphi\right),
$$

where

$$
\alpha(\varphi)=\frac{1}{2}\left(c_{0} \cos \varphi+\left(1-c_{0}\right) \sin \varphi\right)
$$

Proof. The key point is that for $g_{0}=\frac{1}{2}$ the nonlinearity is a scalar factor in front of a fixed matrix:

$$
N(t+1)=\sqrt{R_{0}} e^{-\frac{1}{2} I(t)}\left(\begin{array}{ll}
0 & 1 \\
1 & 0
\end{array}\right) N(t)
$$

Now note that the matrix $\left(\begin{array}{ll}0 & 1 \\ 1 & 0\end{array}\right)$ maps the line through the origin with angle $\varphi$ into the line with angle $\frac{\pi}{2}-\varphi$. If $\left(\begin{array}{l}N_{0} \\ N_{1}\end{array}\right)=r\left(\begin{array}{c}\cos \varphi \\ \sin \varphi\end{array}\right)$ then the image point has radius $\sqrt{R_{0}} r e^{-\alpha(\varphi) r}$ with $\alpha(\varphi)$ as above.

Corollary 5.2. Let $g_{0}=\frac{1}{2}$. The line with angle $\frac{\pi}{4}$ is invariant. Every other straight line through the origin is mapped into itself by the second iterate. The position along the line with angle $\varphi$ evolves under the second iterate according to the one-dimensional map

$$
r \mapsto R_{0} r e^{-\alpha(\varphi) r-\sqrt{R_{0}} \alpha\left(\frac{\pi}{2}-\varphi\right) r e^{-\alpha(\varphi) r}} .
$$

Nontrivial fixed points of (5.3) correspond, for $\varphi \neq \frac{\pi}{4}$, to 2-cycles of (2.1). These lie on a curve in $\left(N_{0}, N_{1}\right)$-space which, in polar coordinates, is determined by the equation

$$
r\left(\alpha(\varphi)+\sqrt{R_{0}} \alpha\left(\frac{\pi}{2}-\varphi\right) e^{-\alpha(\varphi) r}\right)=\ln R_{0} .
$$

The point $\varphi=\frac{\pi}{4}, r=\sqrt{2} \ln R_{0}$ on this curve corresponds to the steady state (3.1).

We can now explain the bifurcation that happens when (3.2) gets violated by $g_{0}$ moving through the value $\frac{1}{2}$. First, note that equality in (3.2) corresponds to an eigenvalue -1 for the linearization, so, in principle, to period-doubling. Here we find a "vertical bifurcation" variant of period-doubling, in the sense that exactly for the bifurcation value $g_{0}=\frac{1}{2}$ there is a one-parameter family of 2-cycles determined, implicitly, by (5.4). (In [4] Cushing and Li deduce a result that is similar to our Proposition 9.1 below and they say on p. 513: "... what the nature of the bifurcation phenomena at both the equilibria and the synchronous 2-cycles is, are interesting mathematical questions which we leave unexplored". No doubt the vertical bifurcations that we find here and in the next section also occur in their model and, accordingly, we think that the questions are now answered.) If we fix $\varphi$ there are generically, as we shall explain in detail in Section 7, either one or three 

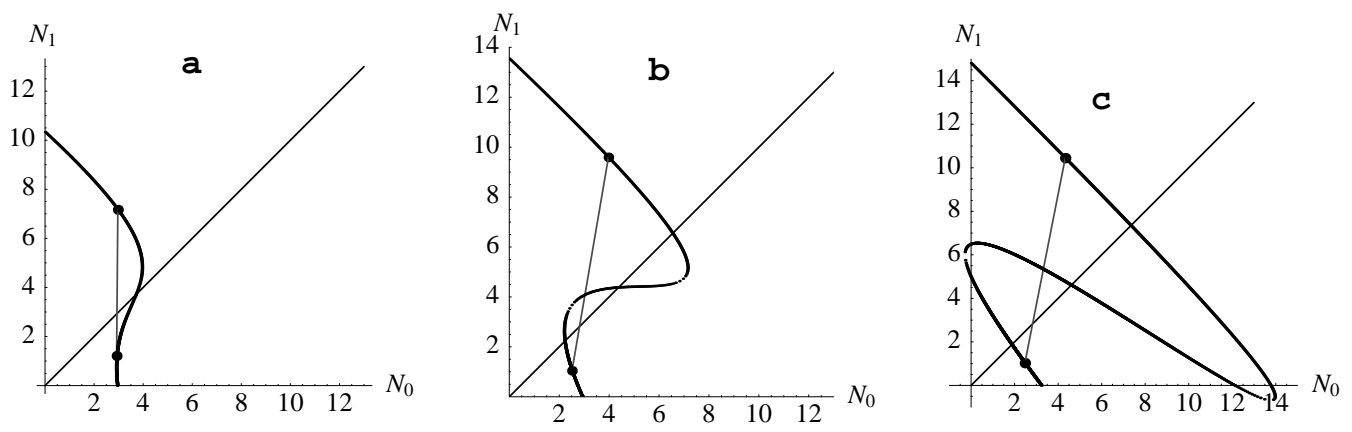

Figure 6: Three kinds of patterns of 2-cycles for the degenerate case $g_{0}=\frac{1}{2}$. a: $R_{0}=40<e^{4}, c_{0}=0.4$, b: $R_{0}=80>e^{4}, c_{0}=0.45, \mathrm{c}: R_{0}=100>e^{4}, c_{0}=0.483$.

solutions of equation (5.4) for $r$. This tells us how the curve defined by (5.4) can, and cannot, fold in $\left(N_{0}, N_{1}\right)$-space. The three possibilities for the global behaviour of the curve are illustrated in Figure 6.

\section{The special case $c_{0}=\frac{R_{0}^{1 / 2-g_{0}}}{1+R_{0}^{1 / 2-g_{0}}}$ of "uniform impact" .}

We next concentrate on the second surface in parameter space that was excluded in Theorem 4.2 (and, actually, also in Theorem 4.1) and which corresponds to the second factor at the left hand side of the condition (3.2) being zero. We call this the "case of uniform impact" for the following reason. Consider a newborn individual under the constant environmental condition $\bar{I}=\ln R_{0}$. Its impact while belonging to age class 0 is given by $c_{0}$. Its expected impact one year later while belonging to age class 1 equals $c_{1} \sqrt{R_{0}} e^{-g_{0} \bar{I}}$. These two quantities are equal if and only if $c_{0}=\frac{R_{0}^{\frac{1}{2}-g_{0}}}{1+R_{0}^{\frac{1}{2}-g_{0}}}$. In this case too we shall find a vertical period-doubling bifurcation. Remarkably, now the one-dimensional continuum of 2-cycles forms a straight line in $\left(N_{0}, N_{1}\right)$-space.

Theorem 6.1. Let $c_{0}=\frac{R_{0}^{\frac{1}{2}-g_{0}}}{1+R_{0}^{\frac{1}{2}-g_{0}}}$ (or, equivalently, $c_{0}=c_{1} \sqrt{R_{0}} e^{-g_{0} \bar{I}}$ ), then the line $\left\{N: I=c \cdot N=\ln R_{0}\right\}$ is invariant.

Proof. We have to check that $c_{0} \sqrt{R_{0}} e^{-g_{1} \ln R_{0}} N_{1}+c_{1} \sqrt{R_{0}} e^{-g_{0} \ln R_{0}} N_{0}=\ln R_{0}$, whenever $c_{0} N_{0}+c_{1} N_{1}=\ln R_{0}$. When $c_{0}=c_{1} \sqrt{R_{0}} e^{-g_{0} \ln R_{0}}$ this follows at once.

Corollary 6.2. On this invariant line the dynamics are given by $N(t+1)=$ $L(\bar{I}) N(t)$ and consequently every point is periodic with period two (with the steady state as the only exception, this is also the minimal period).

Theorem 6.3. At least for $1<R_{0}<e^{2}$ the invariant line is an attractor.

Proof. Every point of the line is a fixed point for the second iterate of the map (2.1). So the Jacobi matrix of this second iterate has an eigenvalue one in every point of the line. Our task is to compute the second eigenvalue (let's call it $\lambda$ ) and to check that it is less than one in absolute value for $1<R_{0}<e^{2}$. 
The second eigenvalue $\lambda$ equals the determinant of the Jacobi matrix. The Jacobi matrix is the product of two Jacobi matrices of the map (2.1), one in a point $\left(N_{0}, N_{1}\right)^{T}$ on the invariant line and the other in the image (under (2.1)) point $\sqrt{R_{0}}\left(e^{-g_{1} \bar{I}} N_{1}, e^{-g_{0} \bar{I}} N_{0}\right)^{T}$ which, by invariance, is on the same line. So the second eigenvalue $\lambda$ equals the product of two determinants of the Jacobi matrix of (2.1) corresponding to two such points.

As in the proof of Theorem 3.1 one derives that the Jacobi matrix of (2.1) in a point $\left(N_{0}, N_{1}\right)^{T}$ on the invariant line is given by

$$
\left(\begin{array}{cc}
-g_{1} c_{0} \sqrt{R_{0}} e^{-g_{1} \bar{I}} N_{1} & -g_{1} c_{1} \sqrt{R_{0}} e^{-g_{1} \bar{I}} N_{1}+\sqrt{R_{0}} e^{-g_{1} \bar{I}} \\
-g_{0} c_{0} \sqrt{R_{0}} e^{-g_{0} \bar{I}} N_{0}+\sqrt{R_{0}} e^{-g_{0} \bar{I}} & -g_{0} c_{1} \sqrt{R_{0}} e^{-g_{0} \bar{I}} N_{0}
\end{array}\right) .
$$

Its determinant equals

$$
g_{0} c_{0} N_{0}+g_{1} c_{1} N_{1}-1
$$

and accordingly the determinant in the image point equals

$$
g_{0} c_{0} \sqrt{R_{0}} e^{-g_{1} \bar{I}} N_{1}+g_{1} c_{1} \sqrt{R_{0}} e^{-g_{0} \bar{I}} N_{0}-1=g_{0} c_{1} N_{1}+g_{1} c_{0} N_{0}-1
$$

So the second eigenvalue, being the product of these two determinants, is given by the formula

$$
\lambda=1-\ln R_{0}+g_{0} g_{1}\left(c_{0}^{2} N_{0}^{2}+c_{1}^{2} N_{1}^{2}\right)+\left(g_{0}^{2}+g_{1}^{2}\right) c_{0} c_{1} N_{0} N_{1} .
$$

Since $1=\left(g_{0}+g_{1}\right)^{2}=g_{0}^{2}+g_{1}^{2}+2 g_{0} g_{1}$ we may rewrite the expression at the right hand side as follows

$$
\lambda=1-\ln R_{0}+c_{0} c_{1} N_{0} N_{1}+g_{0} g_{1}\left(c_{0} N_{0}-c_{1} N_{1}\right)^{2} .
$$

Hence $\lambda \geq 1-\ln R_{0}$ and consequently $\lambda>-1$ for $R_{0}<e^{2}$.

Since $g_{0} g_{1}=g_{0}\left(1-g_{0}\right) \leq \frac{1}{4}$ and, on the invariant line

$$
\frac{1}{4}\left(c_{0} N_{0}-c_{1} N_{1}\right)^{2}+c_{0} c_{1} N_{0} N_{1} \leq \frac{1}{4}\left(c_{0} N_{0}+c_{1} N_{1}\right)^{2}=\frac{1}{4}\left(\ln R_{0}\right)^{2}
$$

we have $\lambda<1$ whenever $\frac{1}{4}\left(\ln R_{0}\right)^{2}-\ln R_{0}<0$ or, equivalently (for $R_{0}>1$ ), whenever $R_{0}<e^{4}$.

Note that the condition on $R_{0}$ is sharp if we want uniformity in $g_{0}$ and $c_{0}$. (Indeed, take $g_{0}=0$ and $c_{0}=0$.)

In conclusion of this section, we note that both factors in (3.2) vanish at the line in parameter space determined by the two conditions $g_{0}=\frac{1}{2}, c_{0}=\frac{1}{2}$. The easiest way to analyse the dynamics for this very special case is to trace the additional features in the results of Section 5 that derive from putting $c_{0}=\frac{1}{2}$ and to fit these in with the results in this section so far.

For $c_{0}=\frac{1}{2}$ we have (cf. 5.2)

$$
\alpha(\varphi)=\frac{1}{4}(\cos \varphi+\sin \varphi)=\alpha\left(\frac{\pi}{2}-\varphi\right) .
$$

Consequently, the family of maps (5.3), parametrised by $\varphi$, has fixed points

$$
r=\frac{1}{\alpha(\varphi)} \frac{\ln R_{0}}{2}
$$

which form the straight line $N_{0}+N_{1}=2 \ln R_{0}$. In fact, the maps (5.3) differ from each other only by a scaling of $r$. Indeed, by scaling $r$ with $\alpha$ we obtain the map

$$
r \mapsto R_{0} r e^{-r-\sqrt{R_{0}} r e^{-r}}
$$

which does not depend on $\varphi$. 


\section{Single year class dynamics.}

The "full life cycle" map $F$ is, by definition, obtained by applying the "one year ahead" map $N \mapsto L(I) N$ (introduced in (2.1)) twice. To represent $F$ explicitly, it is convenient to now call $I$ by the name $I_{0}$, so

$$
I_{0}=c \cdot N=c_{0} N_{0}+c_{1} N_{1}
$$

and to introduce the environmental condition in the next year as

$$
I_{1}=\sqrt{R_{0}}\left\{c_{0} e^{-g_{1} I_{0}} N_{1}+c_{1} e^{-g_{0} I_{0}} N_{0}\right\}
$$

(note that $I_{1}$ depends nonlinearly on $N$ ). With these notations available we can write

$$
F(N)=L\left(I_{1}\right) L\left(I_{0}\right) N=R_{0}\left(\begin{array}{cc}
e^{-g_{0} I_{0}-g_{1} I_{1}} & 0 \\
0 & e^{-g_{0} I_{1}-g_{1} I_{0}}
\end{array}\right)\left(\begin{array}{l}
N_{0} \\
N_{1}
\end{array}\right) .
$$

Since the matrix is diagonal, the coordinate axes are invariant under $F$. In this section we investigate the dynamics of iterating the restriction of $F$ to one such axis, which biologically corresponds to the situation that one of the two year classes is missing.

It is irrelevant to which axis we restrict $F$. This is biologically evident, but the mathematical underpinning is of some interest. Denote the $N_{0}$-axis by $X_{0}$ and the $N_{1}$-axis by $X_{1}$. The map $N \mapsto L(I) N$ maps $X_{0}$ into $X_{1}$ and, likewise, $X_{1}$ into $X_{0}$. Let $\hat{f}: X_{0} \rightarrow X_{1}$ and $\tilde{f}: X_{1} \rightarrow X_{0}$ denote the corresponding restrictions of $N \mapsto L(I) N$. Then $\left.F\right|_{X_{0}}=\tilde{f} \circ \hat{f}$ and $\left.F\right|_{X_{1}}=\hat{f} \circ \tilde{f}$. So, $\left.F\right|_{X_{0}} \circ \tilde{f}=\left.\tilde{f} \circ F\right|_{X_{1}}$ and $\left.\hat{f} \circ F\right|_{X_{0}}=\left.F\right|_{X_{1}} \circ \hat{f}$. By induction it follows that an orbit of $\left.F\right|_{X_{1}}$ is mapped, by $\tilde{f}$, to an orbit of $\left.F\right|_{X_{0}}$ while, conversely, an orbit of $\left.F\right|_{X_{0}}$ is mapped by $\hat{f}$ to an orbit of $\left.F\right|_{X_{1}}$. (And if we map an orbit of $\left.F\right|_{X_{1}}$ first to $X_{0}$ by $\tilde{f}$ and then back to $X_{1}$ by $\hat{f}$, every point is mapped to the next point on the same orbit.) So the phase portraits (i.e. the qualitative orbit structures) of $\left.F\right|_{X_{0}}$ and $\left.F\right|_{X_{1}}$ are identical. Note that neither $\hat{f}$ nor $\tilde{f}$ is an homeomorphism (as the graphs are humped and, consequently, the functions cannot be inverted) so this "equivalence" of $\left.F\right|_{X_{0}}$ and $\left.F\right|_{X_{1}}$ is not the standard one from the theory of dynamical systems.

Writing $y$ rather than $N_{0}$, the map $\left.F\right|_{X_{0}}$ is given explicitly by

$$
y \mapsto R_{0} y e^{-\left(g_{0} c_{0}+g_{1} c_{1} \sqrt{R_{0}} e^{-g_{0} c_{0} y}\right) y} .
$$

If either $g_{0} c_{0}=0$ or $g_{1} c_{1}=0$ this is the well-studied (and consequently wellunderstood) Ricker map. For $g_{0} c_{0} \neq 0$, introduce the scaled variable $x=g_{0} c_{0} y$ to transform (7.4) into the two (rather than three) parameter family of one-dimensional maps:

$$
x \mapsto R_{0} x e^{-h(x, p)},
$$

where

$$
h(x, p)=x\left(1+p e^{-x}\right)
$$

with

$$
p=\sqrt{R_{0}} \frac{g_{1} c_{1}}{g_{0} c_{0}} .
$$

The map (7.5) should be called the "single year class, full life cycle" map, but we shall write SYC-map for short. (Note that for $p=0$ this is the Ricker map.)

Definition 7.1. We call nontrivial periodic points of the $S Y C$-map $S Y C$ periodic points and nontrivial fixed points $S Y C$ fixed points. 
A SYC fixed point $\bar{x}$ corresponds to a 2-periodic point $\left(\frac{\bar{x}}{g_{0} c_{0}}, 0\right)^{T}$ of the original map (2.1). This point lies on the $N_{0}$-axis and its image $\left(0, \frac{\sqrt{R_{0}}}{g_{0} c_{0}} e^{-\bar{x}}\right)^{T}$ lies, of course, on the $N_{1}$-axis, so we can consider a SYC fixed point as a 2-cycle of the original map which takes the values at the axes. We therefore also call it a boundary 2-cycle.

Similarly, a SYC $m$-periodic point corresponds to a boundary $2 m$-cycle of the original map.

The SYC-map inherits the symmetry of the original map (2.1).

Proposition 7.2. For fixed $R_{0}$, the dynamics generated by (7.5) does not change if we replace $p$ by $p^{-1} R_{0}$.

Proof. Essentially, this is a corollary of Proposition 2.1. But a direct proof can easily be given. This direct proof is, in fact, a more explicit version of the equivalence proof of $\left.F\right|_{X_{0}}$ and $\left.F\right|_{X_{1}}$ above, but now for scaled versions of these maps.

Fix $R_{0}$ and write

$$
\begin{aligned}
& f(x, p)=R_{0} x e^{-h(x, p)} \\
& \psi(x, p)=p x e^{-x}
\end{aligned}
$$

then (as one easily can check)

$$
\psi(f(x, p), p)=f\left(\psi(x, p), p^{-1} R_{0}\right) .
$$

Let, for given $x_{0}$, the sequence $\left\{x_{n}\right\}$ be defined recursively by

$$
x_{n+1}=f\left(x_{n}, p\right) .
$$

Similarly, for given $y_{0}$, let $\left\{y_{n}\right\}$ be defined by

$$
y_{n+1}=f\left(y_{n}, p^{-1} R_{0}\right) .
$$

If $y_{0}=\psi\left(x_{0}, p\right)$ then, by induction, $y_{n}=\psi\left(x_{n}, p\right)$. And, similarly, if $x_{0}=$ $\psi\left(y_{0}, p^{-1} R_{0}\right)$ then $x_{n}=\psi\left(y_{n}, p^{-1} R_{0}\right)$. So $\psi$ maps orbits to orbits, the direction being determined by the choice of parameter.

Corollary 7.3. The bifurcation diagram in the $\left(R_{0}, p\right)$ parameter plane is symmetric, that is, invariant under the reflection

$$
\left(R_{0}, p\right) \mapsto\left(R_{0}, p^{-1} R_{0}\right)
$$

(which has the curve of fixed points $p=\sqrt{R_{0}}$ ).

We now summarise our understanding of the dynamics generated by the SYCmap (7.5) in the form of the bifurcation diagram Fig. 7 amplified by a sequence of explanatory remarks. (The same bifurcation diagram but less detailed and in another parameter plane is presented in [14].) A separate manuscript [6] by one of us, giving precise formulations and detailed proofs (based on general theory as, for instance, presented in the books $[7,11,12])$ is in preparation.

The remarks that now follow describe the behaviour of iterates of the SYC-map in different regions of the $\left(R_{0}, p\right)$-parameter plane as well as the bifurcations that occur on the curves that separate these regions from each other.

- In the region $R_{0} \leq 1$ the SYC-map has a unique (and trivial) fixed point which is globally stable. The line $R_{0}=1$ (not plotted in Figure 7 ) corresponds to a transcritical bifurcation leading to the appearance of a nontrivial fixed point. 


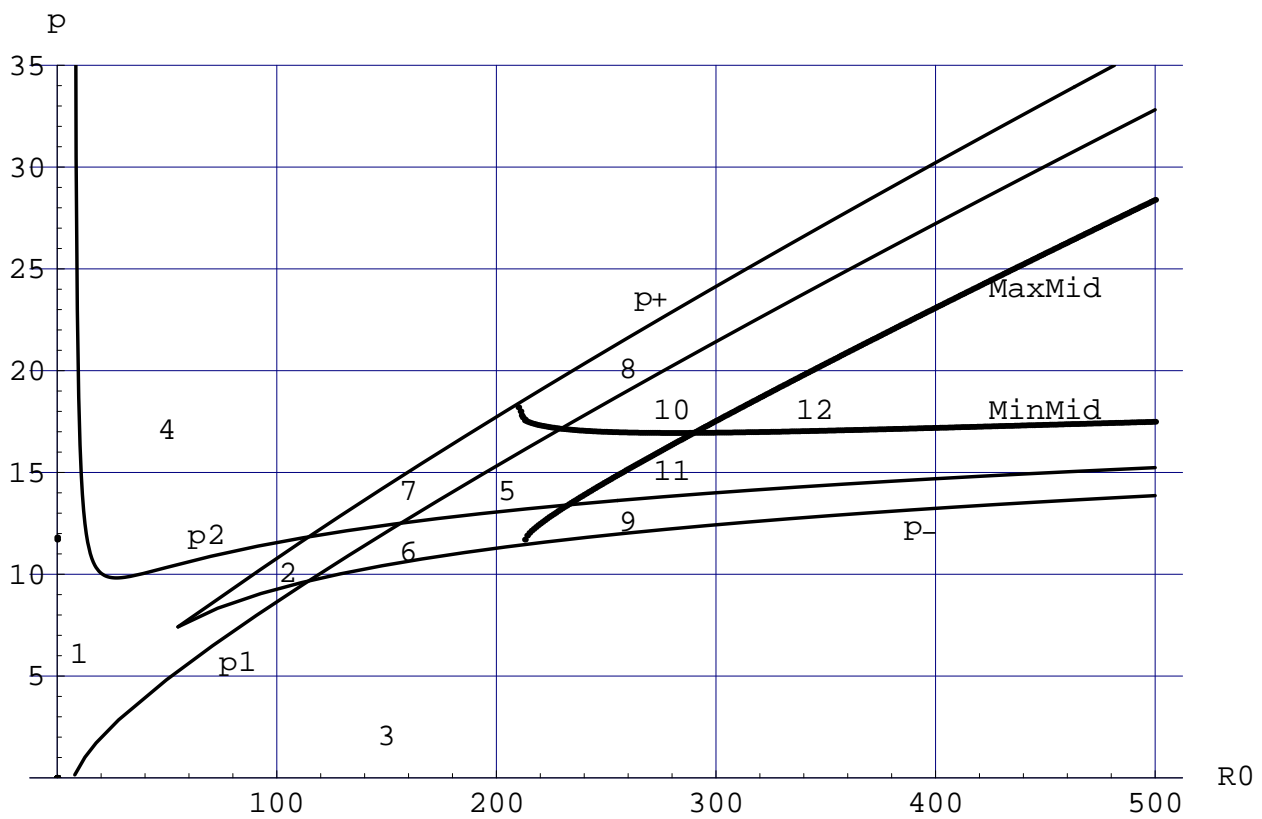

Figure 7: The bifurcation diagram of the SYC-map (7.5).

- For $R_{0}>1$ the SYC-map can have from one to three nontrivial fixed points. The region of three fixed point is the interior of the wedge which is bounded by the curves $p=p_{ \pm}\left(R_{0}\right)$, where

$$
\begin{aligned}
& p_{ \pm}\left(R_{0}\right)=e^{x_{ \pm}}\left(\frac{\ln R_{0}}{x_{ \pm}}-1\right) \\
& x_{ \pm}=\frac{\ln R_{0} \pm \sqrt{\ln ^{2} R_{0}-4 \ln R_{0}}}{2} .
\end{aligned}
$$

These curves correspond to a fold bifurcation resulting in the (dis)appearance of a couple of fixed points; one unstable and one stable. Outside this wedge and, in particular, for $R_{0} \leq e^{4}$ the SYC-map has a unique nontrivial fixed point.

- Consider the region where the SYC-map has a unique nontrivial fixed point. It is divided into three regions: 1,3 and 4 . In region 1 , which includes, in particular, the whole strip $1<R_{0} \leq e^{2}$, the fixed point is globally stable. The curves $p=p_{1,2}\left(R_{0}\right)$, where

$$
\begin{aligned}
& p_{1,2}\left(R_{0}\right)=e^{x_{1,2}}\left(\frac{\ln R_{0}}{x_{1,2}}-1\right) \\
& x_{1,2}=\frac{\ln R_{0} \pm \sqrt{\ln ^{2} R_{0}-4 \ln R_{0}+8}}{2}
\end{aligned}
$$

correspond to a period-doubling bifurcation. At these curves the fixed point loses its stability and a stable 2-cycle appears supercritically. 
In the regions 3 and 4 the fixed point is unstable. Numerically we "observe" a cascade of period-doubling curves. If we follow a one-dimensional path in parameter space, it depends on the way the path intersects the family of period-doubling curves what one observes. In particular, a sequence of period doublings may very well be followed by a sequence of period halvings.

- Consider the interior of the wedge in which the SYC-map has three nontrivial fixed points. The middle one of these fixed points is always unstable. In region 2 the other two fixed points are stable. The curve $p=p_{1}\left(R_{0}\right)$ corresponds to a period-doubling bifurcation of the upper fixed point. In region 6 we see a period-doubling cascade around this fixed point while the lower (nontrivial) fixed point is stable. Similarly, the curve $p=p_{2}\left(R_{0}\right)$ corresponds to a perioddoubling bifurcation of the lower fixed point. In region 7 a period-doubling cascade arises around this fixed point and the upper fixed point is stable. In region 5 both fixed points are unstable and there is a periodic or a chaotic attractor around each of these points.

- The curves MaxMid and MinMid correspond to a homoclinic bifurcation (see, for example, $[7,5])$. They are given implicitly by

$$
\begin{aligned}
& f(M)=x_{\text {mid }} \\
& f(m)=x_{\text {mid }},
\end{aligned}
$$

where $f$ is the SYC-map (7.5), $x_{m i d}$ is its middle fixed point, $M$ and $m$ are a maximum and a minimum of $f$.

At the curve MaxMid a (chaotic) attractor around the upper fixed point "touches" the basin of a lower attractor. Only the lower attractor "survives" this bifurcation. The conditions that guarantee this are

$$
\begin{aligned}
& f(M)<x_{\text {mid }} \\
& f(m)<x_{\text {mid }}
\end{aligned}
$$

and they are satisfied in the regions 9 and 11. (The difference between 9 and 11 is that in region 9 the lower fixed point is stable, while in region 11 it is unstable.)

If both inequalities above are strictly violated we have the symmetric situation, namely the SYC-map has a unique global attractor which is either the stable upper fixed point (region 8) or a periodic or chaotic attractor around this point (region 10).

As we have already said, the curves MaxMid and MinMid correspond to a homoclinic bifurcation, namely the middle fixed point $x_{m i d}$ has a degenerate homoclinic orbit [7, p. 122-124]. In every neighbourhood of this bifurcation the map has a bifurcation of either fold or period-doubling type. Thus, the homoclinic bifurcation is the accumulation point of simple bifurcations, a rather complicated phenomenon [7].

If the inequalities

$$
\begin{aligned}
& f(M)<x_{\text {mid }} \\
& f(m)>x_{\text {mid }}
\end{aligned}
$$

hold (region 12), then the middle fixed point $x_{m i d}$ admits a nondegenerate homoclicic orbit. By a result in $[7$, Cor. 16.6, p. 125] in every neighbourhood of $x_{\text {mid }}$, there are infinitely many distinct periodic points and corresponding chaotic behaviour. Thus for this parametric region there exists one large chaotic attractor in the system. 


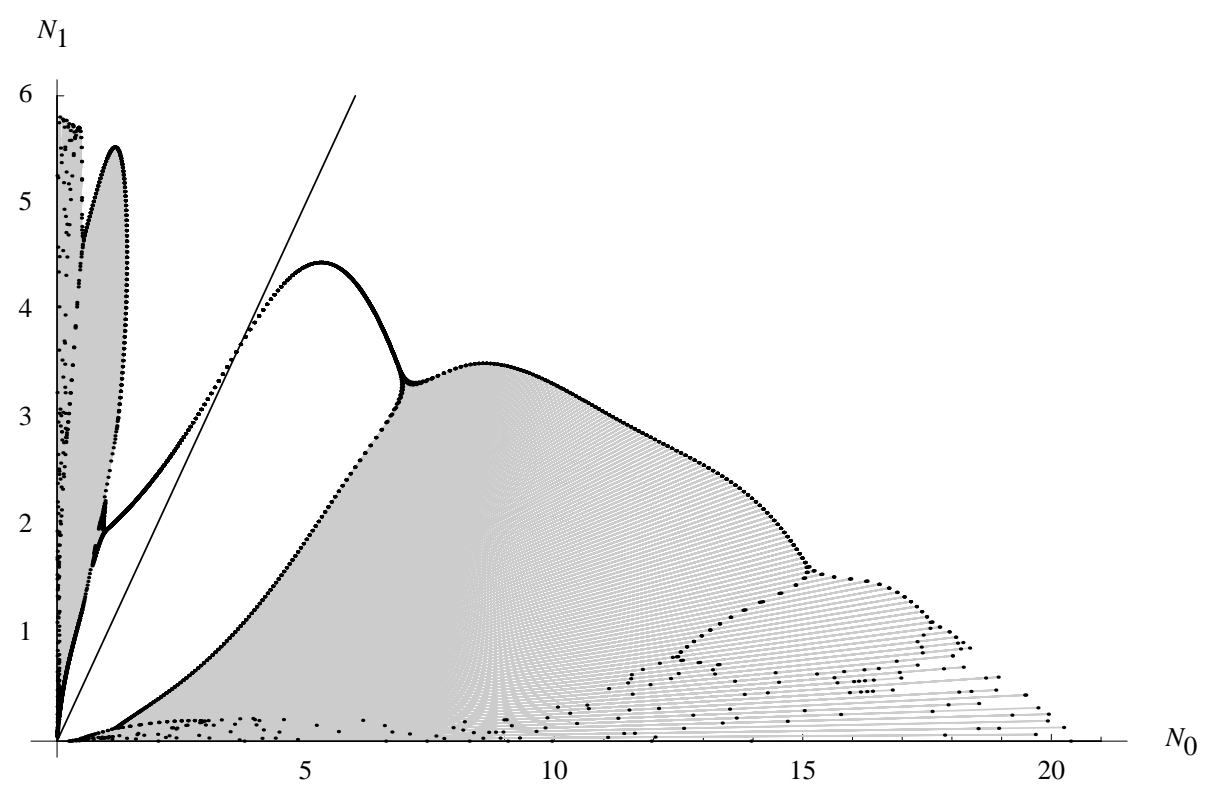

Figure 8: The dynamics of the map (5.3) for different $\varphi$. We see that for $\varphi$ close to the diagonal the map has a stable fixed point while it shows periodic or chaotic behaviour in a neighbourhood of the axes. $R_{0}=40, c_{0}=.8$.

The main conclusions that can be drawn from this description, and which we need for the rest of our analysis, are

- for $1<R_{0} \leq e^{2}$ the SYC-map (7.5) has a unique nontrivial fixed point which is globally stable;

- for $e^{2}<R_{0} \leq e^{4}$ the map has a unique global attractor which is either a fixed point or a periodic or chaotic attractor.

Let us now make a connection between the SYC-map (7.5) and the map (5.3) which describes the dynamics in the special case $g_{0}=\frac{1}{2}$. After the scaling $x=$ $\alpha(\varphi) r$ (for $\alpha(\varphi) \neq 0$ ) the map (5.3) has the same form as the SYC-map (7.5)

$$
x \mapsto R_{0} x e^{-h\left(x, p_{\varphi}\right)}
$$

with the parameter

$$
p_{\varphi}=\sqrt{R_{0}} \frac{\alpha\left(\frac{\pi}{2}-\varphi\right)}{\alpha(\varphi)}=\sqrt{R_{0}} \frac{c_{0} \sin \varphi+c_{1} \cos \varphi}{c_{0} \cos \varphi+c_{1} \sin \varphi} .
$$

(Note that, as should indeed be the case, setting $\varphi=0$ in this expression yields the same result as setting $g_{0}=\frac{1}{2}$ in (7.7).)

Fix $R_{0}$. The parameter $p_{\varphi}$ changes in a monotone fashion from $\sqrt{R_{0}} \frac{c_{1}}{c_{0}}$ to $\sqrt{R_{0}} \frac{c_{0}}{c_{1}}$ as $\varphi$ changes from 0 to $\frac{\pi}{2}$. If $\varphi=\frac{\pi}{4}$, i.e. on the diagonal $N_{0}=N_{1}$, $p_{\varphi}=\sqrt{R_{0}}$ which corresponds to the symmetry axis of the SYC-map. In other words, for different $\varphi$ we find that the map (5.3) shows behaviour corresponding to different values of the parameter $p$ of the SYC-map. We move through the bifurcation diagram (Fig. 7) along a line with fixed $R_{0}$ and with values of $p$ from $\sqrt{R_{0}} \frac{c_{1}}{c_{0}}$ to $\sqrt{R_{0}} \frac{c_{0}}{c_{1}}$.

If $c_{0}=c_{1}=\frac{1}{2}$ the parameter $p_{\varphi}=\sqrt{R_{0}}$ for all values of $\varphi$. Thus the dynamics of the map (5.3) is the same on all the lines through the origin. This is the doubledegenerate case: uniform impact and uniform sensitivity. 
The larger the difference $\left|c_{0}-c_{1}\right|$ the larger the interval over which $p_{\varphi}$ changes. In particular, if either $c_{0}$ or $c_{1}$ is zero, $p_{\varphi}$ moves over the whole interval from 0 to $+\infty$.

We conclude that for $1<R_{0} \leq e^{2}$ the map (5.3) has a unique nontrivial fixed point for all $\varphi$. If $e^{2}<R_{0} \leq e^{4}$ the map (5.3) has a stable fixed point for values of $\varphi$ close to $\frac{\pi}{4}$, while it has a periodic or a chaotic attractor in a neighbourhood of the axes $N_{0}$ and $N_{1}$, i.e. for values of $\varphi$ either close to 0 or $\frac{\pi}{2}$ (Fig. 8).

For $R_{0}>e^{4}$ the situation is more complicated, but we can say that the bistability in the map (5.3) (Fig. 6) occurs for values of $\varphi$ close to the diagonal $\varphi=\frac{\pi}{4}$. It happens because the region of three nontrivial fixed points of the SYC-map lies around the symmetry axis $p=\sqrt{R_{0}}$, which corresponds to the diagonal $\varphi=\frac{\pi}{4}$.

\section{Transversal stability of SYC fixed points.}

Recall from the beginning of Section 7 that the full life cycle map $F$ is given by (7.3), which we here repeat as

$$
F(N)=R_{0}\left(\begin{array}{cc}
e^{-g_{0} I_{0}-g_{1} I_{1}} & 0 \\
0 & e^{-g_{0} I_{1}-g_{1} I_{0}}
\end{array}\right)\left(\begin{array}{c}
N_{0} \\
N_{1}
\end{array}\right) .
$$

Consider a nontrivial fixed point on the $N_{0}$-axis, i.e. a SYC fixed point, then $I_{0}$ and $I_{1}$ are constant, say, respectively, $\bar{I}_{0}$ and $\bar{I}_{1}$, and these quantities are such that

$$
g_{0} \overline{I_{0}}+g_{1} \bar{I}_{1}=\ln R_{0}
$$

If we now introduce the missing year class, i.e. if we make $N_{1}$ slightly positive, then this year class will either grow (and then we say the SYC fixed point is transversally unstable) or decline (in which case we say the SYC fixed point is transversally stable). In fact we have transversal instability if

$$
g_{0} \bar{I}_{1}+g_{1} \bar{I}_{0}<\ln R_{0}
$$

and stability if the reverse inequality holds. Using (8.2) we rewrite (8.3) in the form

$$
\left(g_{0}-g_{1}\right)\left(\bar{I}_{1}-\bar{I}_{0}\right)<0 \text {. }
$$

Using subsequently that $g_{1}=1-g_{0}, \bar{I}_{0}=c_{0} \bar{N}_{0}, \bar{I}_{1}=\sqrt{R_{0}} c_{1} e^{-g_{0} \bar{I}_{0}} \bar{N}_{0}, c_{1}=1-c_{0}$, we find that (8.4) is equivalent to

$$
\left(g_{0}-\frac{1}{2}\right)\left(c_{0}-\frac{\sqrt{R_{0}} e^{-g_{0} \bar{I}_{0}}}{1+\sqrt{R_{0}} e^{-g_{0} \bar{I}_{0}}}\right)>0,
$$

where, for $c_{0}>0, \bar{I}_{0}$ is implicitly determined by the equation

$$
g_{0} \bar{I}_{0}+g_{1} c_{1} \sqrt{R_{0}} e^{-g_{0} \bar{I}_{0}} \frac{\bar{I}_{0}}{c_{0}}=\ln R_{0}
$$

while $\bar{I}_{0}=0$ for $c_{0}=0$.

Comparison of (8.5) and (3.2) reveals a striking resemblance which, as the next result shows, can even be strengthened.

Lemma 8.1. Assume $1<R_{0}<e^{4}$. Then

$$
\operatorname{sign}\left(c_{0}-\frac{R_{0}^{\frac{1}{2}-g_{0}}}{1+R_{0}^{\frac{1}{2}-g_{0}}}\right)=\operatorname{sign}\left(c_{0}-\frac{\sqrt{R_{0}} e^{-g_{0} \bar{I}_{0}}}{1+\sqrt{R_{0}} e^{-g_{0} \bar{I}_{0}}}\right),
$$

where, for $c_{0}>0, \bar{I}_{0}$ is the unique solution of (8.6) (and $\bar{I}_{0}=0$ for $c_{0}=0$ ). 
Proof. For $c_{0}=0$ both conditions are violated. Assume $c_{0}>0$. According to the results reported in Section 7 the SYC-map has, for $1<R_{0}<e^{4}$, a unique nontrivial steady state and consequently equation (8.6) too has a unique positive solution $\bar{I}_{0}$.

A first claim is that

$$
\operatorname{sign}\left(\ln R_{0}-\bar{I}_{0}\right)=\operatorname{sign}\left(\frac{R_{0}^{\frac{1}{2}-g_{0}}}{1+R_{0}^{\frac{1}{2}-g_{0}}}-c_{0}\right) .
$$

To substantiate this claim we introduce the function $G$ defined by

$$
G(x)=g_{0} x+g_{1} \frac{c_{1}}{c_{0}} \sqrt{R_{0}} x e^{-g_{0} x}
$$

which allows us to write equation (8.6) in the form

$$
G\left(\bar{I}_{0}\right)=\ln R_{0} .
$$

Since $G(0)=0$ and $\bar{I}_{0}$ is unique, we must have that $G(x)<\ln R_{0}$ for $x<\bar{I}_{0}$ and $G(x)>\ln R_{0}$ for $x>\bar{I}_{0}$. Hence

$$
\operatorname{sign}\left(x-\bar{I}_{0}\right)=\operatorname{sign}\left(G(x)-\ln R_{0}\right)
$$

in general, but in particular for $x=\ln R_{0}$. Now

$$
\begin{aligned}
& G\left(\ln R_{0}\right)-\ln R_{0}=g_{1}\left(\frac{c_{1}}{c_{0}} R_{0}^{\frac{1}{2}-g_{0}}-1\right) \ln R_{0}=g_{1}\left(\frac{R_{0}^{\frac{1}{2}-g_{0}}}{c_{0}}-\left(1+R_{0}^{\frac{1}{2}-g_{0}}\right)\right) \ln R_{0}= \\
& \frac{g_{1}}{c_{0}}\left(1+R_{0}^{\frac{1}{2}-g_{0}}\right)\left(\frac{R_{0}^{\frac{1}{2}-g_{0}}}{1+R_{0}^{\frac{1}{2}-g_{0}}}-c_{0}\right) \ln R_{0}
\end{aligned}
$$

which shows that the claim is warranted.

A second claim is that

$$
\operatorname{sign}\left(\ln R_{0}-\bar{I}_{0}\right)=\operatorname{sign}\left(\frac{\sqrt{R_{0}} e^{-g_{0} \bar{I}_{0}}}{1+\sqrt{R_{0}} e^{-g_{0} \bar{I}_{0}}}-\frac{R_{0}^{\frac{1}{2}-g_{0}}}{1+R_{0}^{\frac{1}{2}-g_{0}}}\right) .
$$

Again we introduce a function, this time $H$ defined by

$$
H(x)=\frac{\sqrt{R_{0}} e^{-g_{0} x}}{1+\sqrt{R_{0}} e^{-g_{0} x}} .
$$

Since $H$ is strictly decreasing we have

$$
\operatorname{sign}\left(\ln R_{0}-\bar{I}_{0}\right)=\operatorname{sign}\left(H\left(\bar{I}_{0}\right)-H\left(\ln R_{0}\right)\right)
$$

which is exactly the claimed identity.

Combining these two claims we arrive at

$$
\operatorname{sign}\left(c_{0}-\frac{R_{0}^{\frac{1}{2}-g_{0}}}{1+R_{0}^{\frac{1}{2}-g_{0}}}\right)=\operatorname{sign}\left(\frac{R_{0}^{\frac{1}{2}-g_{0}}}{1+R_{0}^{\frac{1}{2}-g_{0}}}-\frac{\sqrt{R_{0}} e^{-g_{0} \bar{I}_{0}}}{1+\sqrt{R_{0}} e^{-g_{0} \bar{I}_{0}}}\right) .
$$

Finally, write

$$
c_{0}-\frac{\sqrt{R_{0}} e^{-g_{0} \bar{I}_{0}}}{1+\sqrt{R_{0}} e^{-g_{0} \bar{I}_{0}}}=\left(c_{0}-\frac{R_{0}^{\frac{1}{2}-g_{0}}}{1+R_{0}^{\frac{1}{2}-g_{0}}}\right)+\left(\frac{R_{0}^{\frac{1}{2}-g_{0}}}{1+R_{0}^{\frac{1}{2}-g_{0}}}-\frac{\sqrt{R_{0}} e^{-g_{0} \bar{I}_{0}}}{1+\sqrt{R_{0}} e^{-g_{0} \bar{I}_{0}}}\right)
$$

and observe that the two terms at the right hand side have the same sign which, therefore, is also the sign of the left hand side. 


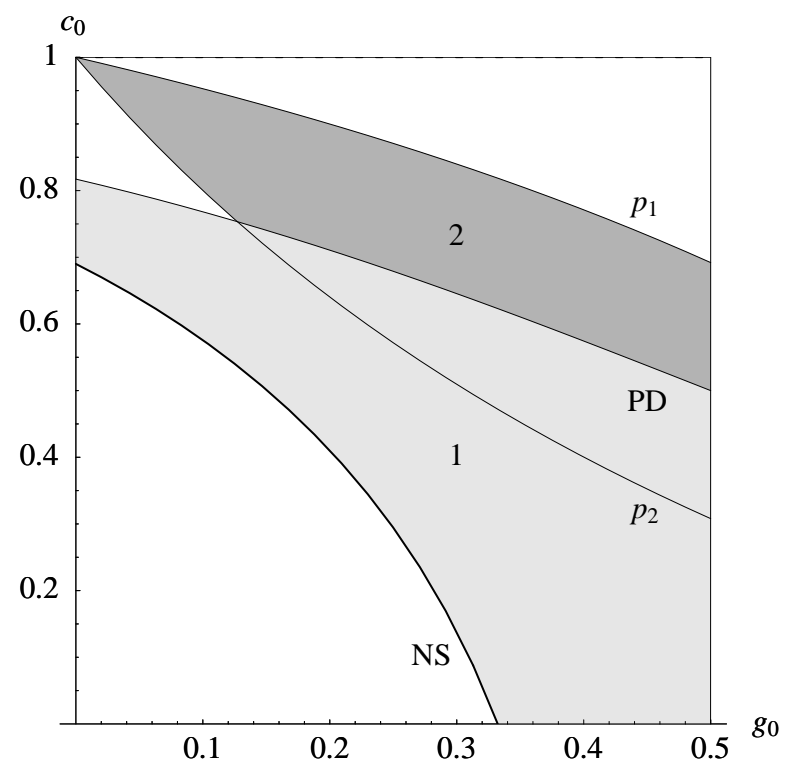

Figure 9: A combined bifurcation diagram depicting the regions of local stability of the internal fixed point and the SYC fixed point. $e^{2}<R_{0}=20<e^{4}$.

Corollary 8.2. Condition (8.5) is satisfied if and only if condition (3.2) is satisfied.

In Section 10 we consider the consequences of this result, with due attention for the biological interpretation. But first we extend the transversal stability analysis to boundary cycles with higher periods.

\section{Transversal stability of SYC periodic points.}

Assume $e^{2}<R_{0}<e^{4}$. We combine the bifurcation diagram for the local behaviour near the internal fixed point (Fig. 5) and the bifurcation diagram of the SYCmap (7.5) (Fig. 7). The result is Figure 9. (We show only half of the diagram because of the reflection symmetry with respect to the point $g_{0}=\frac{1}{2}, c_{0}=\frac{1}{2}$.)

The curves PD (period-doubling bifurcation (3.2)) and NS (Neimark-Sacker bifurcation (3.3)) bound the region of stability of the internal fixed point. The curves $p_{1}$ and $p_{2}$, given by (7.9), correspond to a period-doubling bifurcation of a SYC fixed point. (Recall that for $e^{2}<R_{0}<e^{4}$ the SYC-map has a unique nontrivial fixed point.) To translate the expressions (7.9) for $p_{1,2}$ to $\left(g_{0}, c_{0}\right)$-space, note that a point $\left(p, R_{0}\right)$ corresponds to the curve

$$
c_{0}=\frac{\sqrt{R_{0}}\left(1-g_{0}\right)}{p g_{0}+\sqrt{R_{0}}\left(1-g_{0}\right)},
$$

(so that $c_{0}$ decreases as a function of $g_{0}$ and that the curve goes through the corner points $(0,1)$ and $(1,0))$.

In the preceding section we showed that the curve PD is also the transversal stability boundary for a SYC fixed point. Therefore, in region 1 of the bifurcation diagram an attractor of the system is the stable internal fixed point while in region 2 this fixed point is unstable and the boundary 2-cycle is an attractor. 


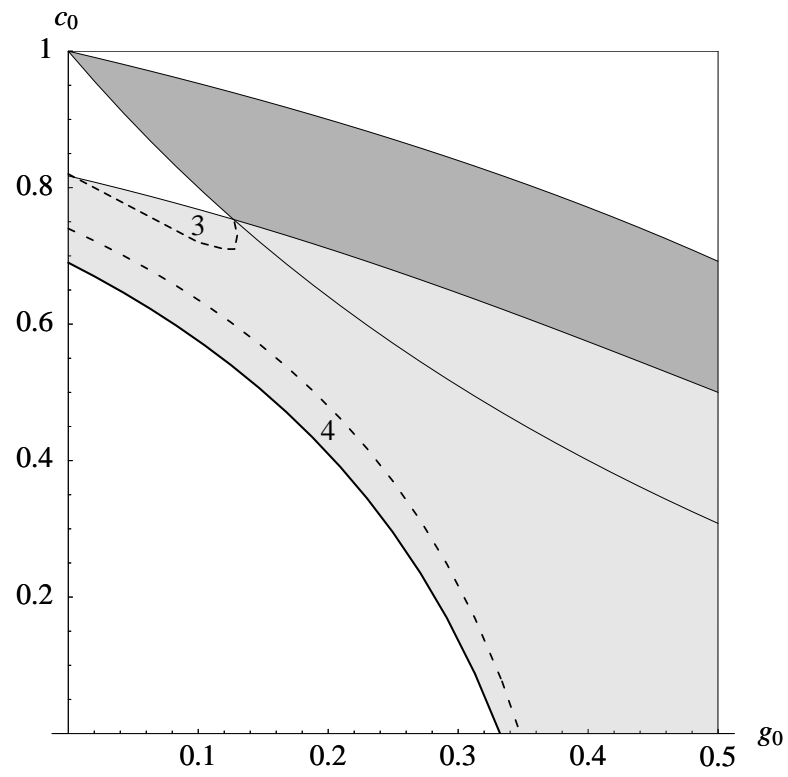

Figure 10: The same bifurcation diagram as in Figure 9 but with regions of bistability indicated: the stable internal fixed point coexists either with a boundary attractor (3) or with a stable internal 3-cycle (4). $e^{2}<R_{0}=20<e^{4}$.

We have proved only local attractivity of the internal fixed point. So we cannot exclude that the system has more than one attractor. Numerical simulations show that there exist at least two parametric regions for $e^{2}<R_{0}<e^{4}$ where the system admits bistability (Fig. 10): the stable internal fixed point coexists either with a boundary attractor (3) (which is not a boundary 2-cycle) or with a stable internal 3 -cycle (4).

The aim of this section is to prove that bistability of the first type is possible, namely that the stable internal fixed point can coexist with a boundary attractor. In particular, we will show that a boundary $2 m$-cycle $(m>1)$ is still transversally stable when a boundary 2-cycle loses its transversal stability. Since the internal fixed point becomes stable when the boundary 2-cycle loses its transversal stability, there exists a parameter region of bistability.

Remark. For $1<R_{0}<e^{2}$ the combined bifurcation diagram looks like the bifurcation diagram for the local stability of the internal steady state (Fig. 1), because for these values of $R_{0}$ the curves $p_{1}$ and $p_{2}$ do not exist and the SYCmap has a unique globally stable nontrivial fixed point. The shaded regions of the bifurcation diagram (Figure 1) correspond to the case when the map (2.1) has an interior fixed point as an attractor, and in the white regions an attractor is a boundary 2-cycle. Numerical simulations indicate that there are no other attractors (bistability does not occur).

Similarly to the previous section we can write down the $m$ th-iterate of the full life cycle map

$$
F^{m}(N)=R_{0}^{m}\left(\begin{array}{cc}
e^{-g_{0} J_{0}-g_{1} J_{1}} & 0 \\
0 & e^{-g_{0} J_{1}-g_{1} J_{0}}
\end{array}\right)\left(\begin{array}{l}
N_{0} \\
N_{1}
\end{array}\right),
$$


where

$$
\begin{aligned}
& J_{0}=\sum_{i=0}^{m-1} I_{2 i} \\
& J_{1}=\sum_{i=0}^{m-1} I_{2 i+1}
\end{aligned}
$$

and the $I$ 's are the values of the environmental variable at the time point as indicated by the index. Fixed points of this map correspond to $m$-periodic points of the full life cycle map $F$ and when they lie on one of the two axes these are SYC $m$-periodic points.

In this section we adopt the convention that we focus our attention on the SYC $m$-periodic points that lie on the $N_{0}$-axis. Consider a nontrivial SYC $m$-periodic point and let $\bar{J}_{0}, \bar{J}_{1}$ denote the corresponding values of $J$, then we should have that

$$
g_{0} \bar{J}_{0}+g_{1} \bar{J}_{1}=m \ln R_{0} .
$$

The $m$-periodic point is transversally unstable if

$$
g_{0} \bar{J}_{1}+g_{1} \bar{J}_{0}<m \ln R_{0} .
$$

Using (9.4) we rewrite (9.5) as

$$
\left(g_{0}-g_{1}\right)\left(\bar{J}_{1}-\bar{J}_{0}\right)<0,
$$

which is mutatis mutandis the same as the inequality (8.4).

We immediately see that the uniform sensitivity condition $g_{0}=g_{1}=\frac{1}{2}$ is again a stability boundary for all SYC periodic points.

Proposition 9.1. Let $g_{0}=0$. Any $S Y C$ periodic point is transversally stable if and only if the $S Y C$ fixed point is transversally stable.

Proof. Consider a SYC periodic point on the $N_{0}$-axis, then $I_{0}=c_{0} N_{0}$ and $I_{1}=$ $c_{1} \sqrt{R_{0}} N_{0}=\frac{c_{1}}{c_{0}} \sqrt{R_{0}} I_{0}$, so $I_{1}<I_{0}$ if and only if $\frac{c_{1}}{c_{0}} \sqrt{R_{0}}<1$. The latter condition is equivalent to $c_{0}>\frac{\sqrt{R_{0}}}{1+\sqrt{R_{0}}}$ which is, for $g_{0}=0$, according to Lemma 8.1 and condition (8.5) exactly the condition for transversal stability of the SYC fixed point. Exactly the same argument shows that $I_{2 i+1}<I_{2 i}$ for arbitrary $i$ if and only if $c_{0}>\frac{\sqrt{R_{0}}}{1+\sqrt{R_{0}}}$. Combining this information with (9.6) we find, via (9.3), the desired conclusion.

Lemma 9.2. Suppose for $g_{0}=0$ and $c_{0}=\frac{\sqrt{R_{0}}}{1+\sqrt{R_{0}}}$ there exists a SYC $\mathrm{m}$ periodic orbit (with $m>1$ ) with "internal" multiplier different from one. Then this $S Y C m$-periodic orbit can be continued for small positive $g_{0}$ along the curve $c_{0}=\frac{R_{0}^{\frac{1}{2}-g_{0}}}{1+R_{0}^{\frac{1}{2}-g_{0}}}$ and it is transversally stable for such $g_{0}$.

Proof. The possibility to continue derives from the assumption that the multiplier associated with the one-dimensional map does not equal one (a manifestation of the general result that hyperbolic fixed points can be continued as a function of a parameter).

It is convenient to forget about the $N_{i}(t)$ and to work with the quantities $I_{j}$ instead. In general we have

$$
I_{2 i+1}=\frac{c_{1}}{c_{0}} \sqrt{R_{0}} I_{2 i} e^{-g_{0} I_{2 i}}
$$


so along the curve we consider we have

$$
I_{2 i+1}=R_{0}^{g_{0}} I_{2 i} e^{-g_{0} I_{2 i}} .
$$

Since $g_{0}-g_{1}<0$ we have, according to (9.6), transversal stability whenever

$$
\sum_{i=0}^{m-1}\left(I_{2 i}-I_{2 i+1}\right)>0 .
$$

According to Proposition 9.1, condition (8.5) and Lemma 8.1, the quantity at the left hand side equals zero for $g_{0}=0$. We therefore intend to prove that the derivative with respect to $g_{0}$ is strictly positive for $g_{0}=0$. Differentiating the identity (9.7) with respect to $g_{0}$ we obtain

$$
\frac{\partial I_{2 i+1}}{\partial g_{0}}=\left(\ln R_{0}\right) R_{0}^{g_{0}} I_{2 i} e^{-g_{0} I_{2 i}}-R_{0}^{g_{0}}\left(I_{2 i}\right)^{2} e^{-g_{0} I_{2 i}}+R_{0}^{g_{0}}\left(1-g_{0}\right) \frac{\partial I_{2 i}}{\partial g_{0}} e^{-g_{0} I_{2 i}}
$$

which, putting $g_{0}=0$, simplifies to

$$
\frac{\partial I_{2 i+1}}{\partial g_{0}}=\left(\ln R_{0}\right) I_{2 i}-\left(I_{2 i}\right)^{2}+\frac{\partial I_{2 i}}{\partial g_{0}}
$$

Hence we have, for $g_{0}=0$,

$$
\frac{\partial}{\partial g_{0}} \sum_{i=0}^{m-1}\left(I_{2 i}-I_{2 i+1}\right)=\sum_{i=0}^{m-1}\left(I_{2 i}^{2}-\ln R_{0} I_{2 i}\right) .
$$

From (9.7) and (9.4), with $g_{0}$ put equal to zero in both, we infer that

$$
\sum_{i=0}^{m-1} I_{2 i}=\sum_{i=0}^{m-1} I_{2 i+1}=m \ln R_{0}
$$

or, in words, the average of the $I_{2 i}$ equals $\ln R_{0}$. Hence

$$
\sum_{i=0}^{m-1}\left(I_{2 i}^{2}-\ln R_{0} I_{2 i}\right)=\sum_{i=0}^{m-1} I_{2 i}^{2}-m\left(\ln R_{0}\right)^{2}=\sum_{i=0}^{m-1}\left(I_{2 i}-\ln R_{0}\right)^{2}
$$

is strictly positive, unless $I_{2 i}=\ln R_{0}$ for all $i$, which implies $m=1$.

Corollary 9.3. Let $e^{2}<R_{0}<e^{4}$. Whenever $R_{0}$ and $m>1$ are such that the Ricker map $x \mapsto R_{0} x e^{-x}$ has a linearly stable $m$-cycle, there exists a $\left(g_{0}, c_{0}\right)$ parameter region, with $\left(g_{0}, c_{0}\right)=\left(0, \frac{\sqrt{R_{0}}}{1+\sqrt{R_{0}}}\right)$ as a boundary point, in which the map (2.1) shows bistability.

Proof. By the symmetry of Proposition 7.2 the SYC-map for $g_{0}=0$ is equivalent to the Ricker map. So, applying Lemma 9.2 we obtain that for small $g_{0}$ and for $c_{0}$ slightly less than $\frac{R_{0}^{\frac{1}{2}-g_{0}}}{1+R_{0}^{\frac{1}{2}-g_{0}}}$ the SYC-map has a stable $m$-cycle which is also transversally stable. For $e^{2}<R_{0}<e^{4}$ the internal fixed point of (2.1) is also stable for (at least some of) these parameter values and the corollary is proved.

Remark. We suppose that for almost all $R_{0}$ in $\left(e^{2}, e^{4}\right)$ there exists $m>1$ such that the Ricker map has a linearly stable $m$-cycle, but we were not able to find a statement to this effect in the literature.

The next section is devoted to the consequences of the results and their interpretation. 


\section{Coexistence or competitive exclusion?}

For $1<R_{0}<e^{2}$ we have that

- the SYC-map (7.5) has a unique globally stable nontrivial fixed point;

- the inequality (3.3) is satisfied for all $\left(g_{0}, c_{0}\right)$ (recall Proposition 3.5.i);

and accordingly we can reformulate Corollary 8.2 as a strict dichotomy.

Theorem 10.1. For $1<R_{0}<e^{2}$ and $g_{0} \neq \frac{1}{2}, c_{0} \neq \frac{R_{0}^{\frac{1}{2}-g_{0}}}{1+R_{0}^{\frac{1}{2}-g_{0}}}$

either the internal steady state (3.1) is asymptotically stable and the SYC fixed point is transversally unstable,

or the internal steady state (3.1) is unstable and the SYC-fixed point is transversally stable.

The first alternative applies when inequality (3.2) holds, the second when (3.2) is violated.

In fact we conjecture that these local stability results govern the global behaviour and that

either the interior of the positive quadrant belongs to the domain of attraction of the internal steady state (3.1),

or the internal steady state is a saddle point with a one-dimensional stable manifold and the positive quadrant is the union of this stable manifold and the domains of attraction of the SYC fixed point at the $N_{0}$-axis and its image at the $N_{1}$-axis.

In biological terms this amounts to

either the two year classes coexist in steady state,

or one outcompetes the other.

The inequality (3.2) determines, in term of the parameters, which of the two alternatives applies. (See Figure 1. The model predicts coexistence of the two year classes in steady state for parameter combinations belonging to the shaded domain, and competitive exclusion for the complement.) In order to interpret the condition we do some rewriting and, in particular, undo the scaling of Section 2. First, note that

$$
\operatorname{sign}\left(g_{0}-\frac{1}{2}\right)=\operatorname{sign}\left(\tilde{g}_{0}-\tilde{g}_{1}\right) \text {. }
$$

Secondly, note that

$$
\begin{aligned}
\operatorname{sign}\left(c_{0}-\frac{R_{0}^{\frac{1}{2}-g_{0}}}{1+R_{0}^{\frac{1}{2}-g_{0}}}\right) & =\operatorname{sign}\left(c_{0}-c_{1} R_{0}^{\frac{1}{2}-g_{0}}\right) \\
& =\operatorname{sign}\left(\tilde{c}_{0}-\tilde{c}_{1} s_{0} R_{0}^{-g_{0}}\right),
\end{aligned}
$$

where $g_{0}=\frac{\tilde{g}_{0}}{\tilde{g}_{0}+\tilde{g}_{1}}$ Combining these two observations we arrive at the following (incomplete) collection of sufficient conditions for competitive exclusion. 


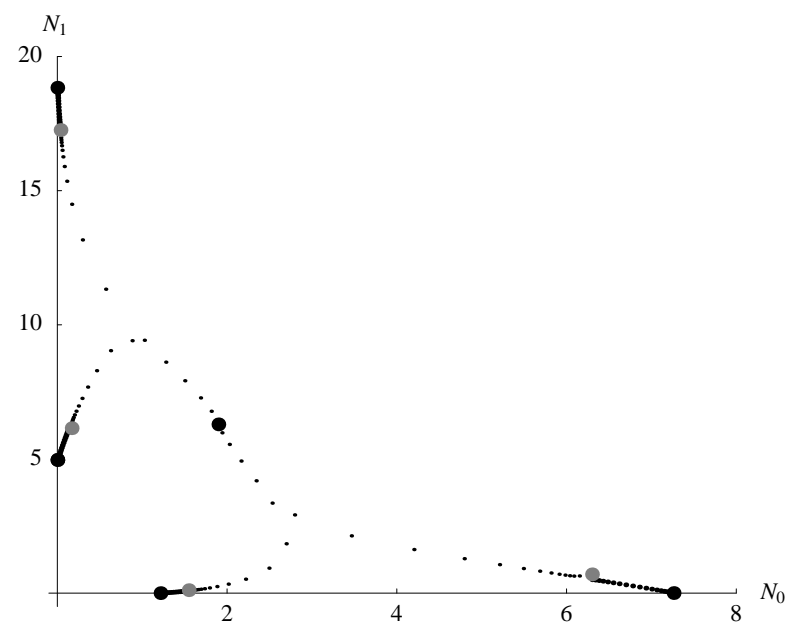

Figure 11: Coexistence of two attractors: the interior steady state and a boundary 4-cycle. An internal saddle 4-cycle is also shown (in gray). The attractors belong to the closure of the unstable manifold of the saddle-cycle. $R_{0}=20, g_{0}=0.1, c_{0}=0.75$.

Theorem 10.2. Competitive exclusion occurs if $\tilde{g}_{1}>\tilde{g}_{0}$, i.e. sensitivity increases with age, while $\tilde{c}_{1}<\tilde{c}_{0}$, i.e. impact decreases with age. It also occurs if sensitivity decreases with age $\left(\tilde{g}_{1}<\tilde{g}_{0}\right)$, while impact increases sufficiently strongly with age $\left(\tilde{c}_{1} s_{0} R_{0}^{-g_{0}}>\tilde{c}_{0}\right)$.

We emphasize one particular aspect of this result: it is not at all unusual, in terms of the parameters, that the attractor is characterized by one of the two year classes being missing.

So far we restricted our attention to $1<R_{0}<e^{2}$ but, in fact, the above picture extends to much of the subset of the parameter space where $e^{2} \leq R_{0}<$ $e^{4}$. In particular, the internal steady state (3.1) and the SYC fixed point cannot simultaneously be stable: if one is stable, the other is not. (In $[4,14]$ this conclusion was derived for similar models.) The difference with the region where $1<R_{0}<e^{2}$ is, first, that for $e^{2} \leq R_{0}<e^{4}$ it becomes possible that both are unstable. Indeed, the extra conditions that matter for $e^{2} \leq R_{0}<e^{4}$ are (3.3) for the interior steady state and $p_{1}<p<p_{2}$, with $p_{1,2}$ defined in (7.9) for the SYC fixed point. In particular, in the parameter region which lies under the NS-curve, given by (3.3), (Fig. 9) the system has an internal attractor which can be either a limit cycle or even a strange attractor. In the white regions above the PD-curve (Fig. 9) the system has a boundary attractor, which is either a boundary $2 m$-cycle with $m>1$ or a boundary chaotic attractor.

But, secondly, as we have shown in Section 9 , for $e^{2}<R_{0}<e^{4}$ two types of bistability are possible in the system (see Fig. 10 for details). Figure 11 presents an example of coexistence of the stable internal fixed point and a stable boundary 4-cycle. So, in other words, the strict dichotomy of Theorem 10.1 does not extend to all of parameter space and it is possible that it depends on the initial conditions whether or not the two year classes will coexist indefinitely.

\section{References}

[1] Baker, J.: Unpublished manuscript. 
[2] Behncke, H.: Periodical cicadas. J. Math. Biol., 40, 413-431 (2000)

[3] Bulmer, M. G.:Periodical Insects. The American Naturalist, 111, 1099-1117 (1977)

[4] Cushing, J. M., Li, J.: Intra-specific competition and density dependent juvenile growth. Bulletin of Mathematical Biology, 54, 503-519 (1992)

[5] Chossat, P., Golubitsky, M.: Symmetry-increasing bifurcation of chaotic attractors. Phys. D 32, no. 3, 423-436 (1988)

[6] Davydova N. V.: Dynamics and bifurcations in families of single year class maps. In preparation.

[7] Devaney, R. L.: An Introduction to Chaotic Dynamical Systems. Second Edition. Addison-Wesley Publishing Company 1989

[8] Diekmann, O., Gils, S. A. van: Difference Equations with Delay. Japan J. Indust. Appl. Math., 17, 73-84 (2000)

[9] Diekmann, O., Gyllenberg, M., Huang, H., Kirkilionis, M., Metz, J. A. J., Thieme, H. R.: On the Formulation and Analysis of General Deterministic Structured Population Models. II. Nonlinear Theory. To appear in J. Math. Biol.

[10] Diekmann, O., Mylius, S. D., Donkelaar, J. R. ten: Saumon à la Kaitala et Getz, sauce hollandaise. Evol. Ecol. Res., 1, 261-275 (1999)

[11] Kuznetsov, Yu. A.: Elements of Applied Bifurcation Theory. Second Edition. Springer-Verlag 1998

[12] Melo, W. de and Strien, S. van.: One-Dimensional Dynamics. Springer-Verlag 1993.

[13] Mylius, S. D., Diekmann, O.: The resident strikes back: invader-induced switching of resident attractor. To appear in J. Theor. Biol.

[14] Nisbet, R. M., Onyiah, L. C.: Population dynamics consequences of competition within and between age classes. J. Math. Biol., 32, 329-344 (1994)

[15] Wikan, A.: Dynamic Consequences of Reproductive Delay in Leslie Matrix Models with Nonlinear Survival Probabilities. Math. Biosciences, 146, 37-62 (1997) 